\title{
Systematic Review \\ A Systematic Review of the Safety of Blocking the IL-1 System in Human Pregnancy
}

\author{
Marie-Eve Brien ${ }^{1}\left(\right.$, , Virginie Gaudreault ${ }^{1}$, Katia Hughes ${ }^{1}$, Dexter J. L. Hayes ${ }^{2}$, Alexander E. P. Heazell ${ }^{2}$ \\ and Sylvie Girard ${ }^{3,4, *}$ \\ 1 Ste-Justine Hospital Research Center, Montreal, QC H3T 1C5, Canada; \\ marie-eve.brien.hsj@ssss.gouv.qc.ca (M.-E.B.); virginie.gaudreault7@gmail.com (V.G.); \\ khugh049@uottawa.ca (K.H.) \\ 2 Maternal and Fetal Health Research Centre, Faculty of Biology, Medicine and Health, \\ University of Manchester, Manchester Academic Health Science Centre, Manchester M13 9PL, UK; \\ dexter.hayes@manchester.ac.uk (D.J.L.H.); alexander.heazell@manchester.ac.uk (A.E.P.H.) \\ 3 Department of Obstetrics and Gynecology, Universite de Montreal, Montreal, QC H3T 1J4, Canada \\ 4 Department of Obstetrics and Gynecology, Department of Immunology, Mayo Clinic, \\ Rochester, MN 55902, USA \\ * Correspondence: girard.sylvie@mayo.edu; Tel.: +1-507-284-0545
}

check for updates

Citation: Brien, M.-E.; Gaudreault, V.; Hughes, K.; Hayes, D.J.L.; Heazell, A.E.P.; Girard, S. A Systematic Review of the Safety of Blocking the IL-1 System in Human Pregnancy. J. Clin. Med. 2022, 11, 225. https:// doi.org/10.3390/jcm11010225

Academic Editor: Yariv Yogev

Received: 10 December 2021

Accepted: 29 December 2021

Published: 31 December 2021

Publisher's Note: MDPI stays neutral with regard to jurisdictional claims in published maps and institutional affiliations.

Copyright: () 2021 by the authors. Licensee MDPI, Basel, Switzerland. This article is an open access article distributed under the terms and conditions of the Creative Commons Attribution (CC BY) license (https:// creativecommons.org/licenses/by/ $4.0 /)$.

\begin{abstract}
Blockade of the interleukin-1 (IL-1) pathway has been used therapeutically in several inflammatory diseases including arthritis and cryopyrin-associated periodic syndrome (CAPS). These conditions frequently affect women of childbearing age and continued usage of IL-1 specific treatments throughout pregnancy has been reported. IL-1 is involved in pregnancy complications and its blockade could have therapeutic potential. We systematically reviewed all reported cases of IL-1 blockade in human pregnancy to assess safety and perinatal outcomes. We searched several databases to find reports of specific blockade of the IL-1 pathway at any stage of pregnancy, excluding broad spectrum or non-specific anti-inflammatory intervention. Our literature search generated 2439 references of which 22 studies included, following extensive review. From these, 88 different pregnancies were assessed. Most (64.8\%) resulted in healthy term deliveries without any obstetri$\mathrm{cal} /$ neonatal complications. Including pregnancy exposed to Anakinra or Canakinumab, 12 (15.0\%) resulted in preterm birth and one stillbirth occurred. Regarding neonatal complications, 2 cases of renal agenesis (2.5\%) were observed, and 6 infants were diagnosed with CAPS (7.5\%). In conclusion, this systematic review describes that IL-1 blockade during pregnancy is not associated with increased adverse perinatal outcomes, considering that treated women all presented an inflammatory disease associated with elevated risk of pregnancy complications.
\end{abstract}

Keywords: IL-1 blockade; anakinra; canakinumab; pregnancy; human; inflammation

\section{Introduction}

Pregnancy complications are often associated with inflammation at the maternal-fetal interface. During pregnancy complication, such as preeclampsia (PE), preterm birth (PTB) and fetal growth restriction (FGR), inflammation can be found in the maternal circulation as well as in the placenta. Uncontrolled inflammation can negatively affect placental function [1-6]. Any alteration in placental function is associated with neonatal complications and altered child development particularly neurodevelopmental delay [7-10]. Therapeutically targeting inflammation in pregnancy has been challenging since inflammatory processes are also involved in physiological pregnancies, especially at the time of implantation and parturition [11-16] and a proinflammatory profile can be observed toward the end of uncomplicated pregnancy [16-18]. Therefore, there is a need to differentiate between physiological and pathological inflammation in order to develop and apply novel anti-inflammatory strategies in the clinical setting. 
Inflammation has been observed at all stages of pregnancy [19-26]. Inflammation can occur in response to bacterial or viral infections (collectively referred to as pathogensassociated molecular patterns-PAMPs), as well as in response to sterile or endogenous mediators, termed damage-associated molecular pattern-DAMPs or alarmins, the latter increasingly associated with pathological pregnancies [27-32]. In order to mitigate the effects of dysregulated inflammation during pregnancy, multiple broad-spectrum antiinflammatory therapies have been used and developed with interesting results [33,34]. Amongst these therapies, corticosteroids and nonsteroidal anti-inflammatory drugs (NSAIDs) are the most prevalent. However, studies have investigated the effect of corticosteroids during pregnancy and found an association between corticosteroid use and pregnancy/neonatal complications [35-38]. In a systematic review, Bandoli G. et al. found little to no association between corticosteroids and adverse pregnancy outcomes [35] whilst other groups reported concerns such as higher rate of cerebral palsy among children who had been exposed to repeated doses of corticosteroids or impaired growth of the lung parenchyma in cases of treatment without premature birth [36-38]. As for NSAIDs, acting through the inhibition of cyclooxygenase enzymes (COX-1 and COX-2), a study by Bérard A. et al., showed elevated risk of prematurity associated with the use of COX-2 inhibitors [39]. However, NSAIDs use during the first trimester of pregnancy was not associated with congenital malformations [40].

In case of pregnancies complicated with infections, antibiotics are often used. However, several studies have shown the detrimental effects of antibiotics on the development of the newborn. In the ORACLE series of clinical studies, the use of some antibiotics in pregnancy has been associated with elevated incidence of neurodevelopmental disorders [41,42]. Others have found increased risk of spontaneous miscarriage [43], without any association with congenital malformation [44]. All these drugs have benefits, but also major risk associated with their use. Since these are not specific and affect several inflammatory pathways at once, it is possible that pathways important in physiological pregnancy are impacted and responsible for the side effects observed. Targeting specific inflammatory mediators/pathways involved in pathological pregnancies could provide an efficient mean to mitigate the negative impact of inflammation, subsequently protecting the placenta and developing fetus, whilst having less deleterious effects.

The interleukin-1 (IL-1) system has been consistently associated with pregnancy complications such as preterm birth, including chorioamnionitis, FGR and PE [1,5,6,32,45-49] as well as high-risk pregnancies with reduced fetal movements [50]. The IL-1 system has been targeted in several animal models of pregnancy complications and blockade of this pathway appears to reduce the incidence of complications and protect the placenta as well as both fetal/neonatal development [4,51-58] and as reviewed previously by us, with emphasis on means of blocking the IL-1 pathway and their mechanisms of action with schematic representation [59]. Aside from studies in animal models, Il-1 blockers have been used for many years to help mitigate/resolve inflammatory conditions in humans [60-63]. The IL-1 receptor antagonist, IL-1Ra, is the most commonly used IL-1 system antagonist and is known under the generic name Anakinra (brand name Kineret). Anakinra has been approved for clinical use for over 20 years and has been commonly used for several chronic inflammatory conditions (such as arthritis and lupus) and in the pediatric population [60,64-68]. Canakinumab, brand name Ilaris, is a monoclonal antibody targeting IL-1 $\beta$ which has been approved for inflammatory condition such as cryopyrin-associated periodic syndromes (CAPS), since $2009[69,70]$. These inflammatory conditions commonly affect women of reproductive age and continued usage of Anakinra and/or Canakinumab during pregnancy has been reported [71-73]. Despite their wide range of beneficial effects, these drugs are not yet approved for use in pregnant women and are used solely when the benefit of continuing the treatment during the pregnancy outweigh the risk.

In light of the important need for targeted anti-inflammatory therapies during pregnancy, the evidence that the IL-1 system is central to both PAMPs and DAMPs-induced inflammation at the maternal-fetal interface, our objective was to perform a systematic 
review of all reports of any specific blockers of the IL-1 system being used during human pregnancy, to assess their potential impact on pregnancy outcome and neonatal health.

\section{Materials and Methods}

The systematic review is reported in accordance with Preferred Reporting Items for Systematic Reviews and Meta-Analyses (PRISMA) guidelines [74]. The review protocol was registered with the International Prospective Register of Systematic Reviews (PROSPERO) on 6 July 2020 (CRD42020197186).

\subsection{Information Sources, Search Strategy and Eligibility Criteria}

Literature searches were conducted in PubMed, EMBASE, MEDLINE, Cochrane Database of Systematic Reviews and Google Scholar. The search was not limited by dates but was limited to titles, abstracts and manuscripts written in English and French (for practical reasons). Reviews were excluded to ensure inclusion of original research only. Abstract from conferences were included as well, unless the same data was published and therefore only the final research article was included to avoid duplication of the same cases. Reference lists of included studies were checked for any other relevant papers. Manuscripts were identified with the search terms 'pregnancy' and 'IL-1 blockage' or 'IL-1 blockade' or 'IL-1 receptor agonist' or 'IL-1ra' or 'Anakinra' or 'Kineret' or 'Rilonacept' or 'Canakinumab' or 'Rytvela'. All searches were completed by 9 July 2021. An example search is included in Data S1.

We included cohort studies (prospective and retrospective), case series and case reports which reported the used of IL-1 blockage during pregnancy. We included all studies involving pregnant individuals who received IL-1 blockage at any stage during their pregnancy.

Our main objective was to document pregnancy outcomes related to treatment (i.e., IL-1 blockade) with IL-1 antagonists during pregnancy. The medical indication for the treatment, chronic inflammatory pathologies diagnosed prior to pregnancy in most cases, was also considered. Data regarding the rates of pregnancy complications (including: congenital anomalies, hypertensive disorders of pregnancy, preterm birth—delivery before 37 weeks of gestation, FGR, neonatal and maternal death) were extracted. We compared all these outcomes to the reported incidence in the general population and population of women with inflammatory pathologies.

\subsection{Data Extraction}

Duplicates were removed, and all citations were screened for relevance using the full abstract and indexing terms. Two out of three reviewers (MEB, VG or KH) had to agree that a study for it to be included, according to the pre-specified inclusion and exclusion criteria. When available, full-length manuscripts were obtained. Two reviewers (MEB and VG) made final inclusions decisions independently and a third reviewer (SG) was consulted to resolve any conflict when necessary.

\subsection{Assessment of Risk of Bias and Methodological Quality}

Included cohort studies were assessed using the Risk of Bias in Non-randomised Studies-of Interventions (ROBINS-I) with 7 domains, since all the studies included in this systematic review were observational. This method categorises each study by low, moderate, serious, critical risk of bias or no information [75]. If a study' risk of bias was categorised as serious or critical, the effect of removing this study was tested and the relevant outcome reported. Individual case reports were assessed using a specific tool to assess the methodological quality of case reports [76]. This assesses 8 characteristics in 4 domains of selection, ascertainment, causality and reporting. 


\subsection{Data Synthesis}

Studies with continuous data (i.e., birthweight and gestational age) were taken to obtain overall means and standard deviations. It was intended to investigate effect of exposure to IL-1 blockade at different times of pregnancy, but data could not be stratified by trimester of exposure since too many data were missing.

\section{Results}

The search strategy (Figure 1) identified 2439 articles. After removing duplicates ( $n=742), 1697$ papers were screened based on their title and abstracts. 1569 papers were excluded based of irrelevant to the question, exposure not during pregnancy and IL-1 blockade effects were not reported. On this basis, resulting in 128 papers for which full text was evaluated. 106 studies were excluded as they were reviews, conference abstract with original article already included, missing information or reports of animal studies, meaning 22 papers were included in the final synthesis. The 22 included studies were 9 case reports, 13 cohort studies (6 retrospective and 7 prospective).

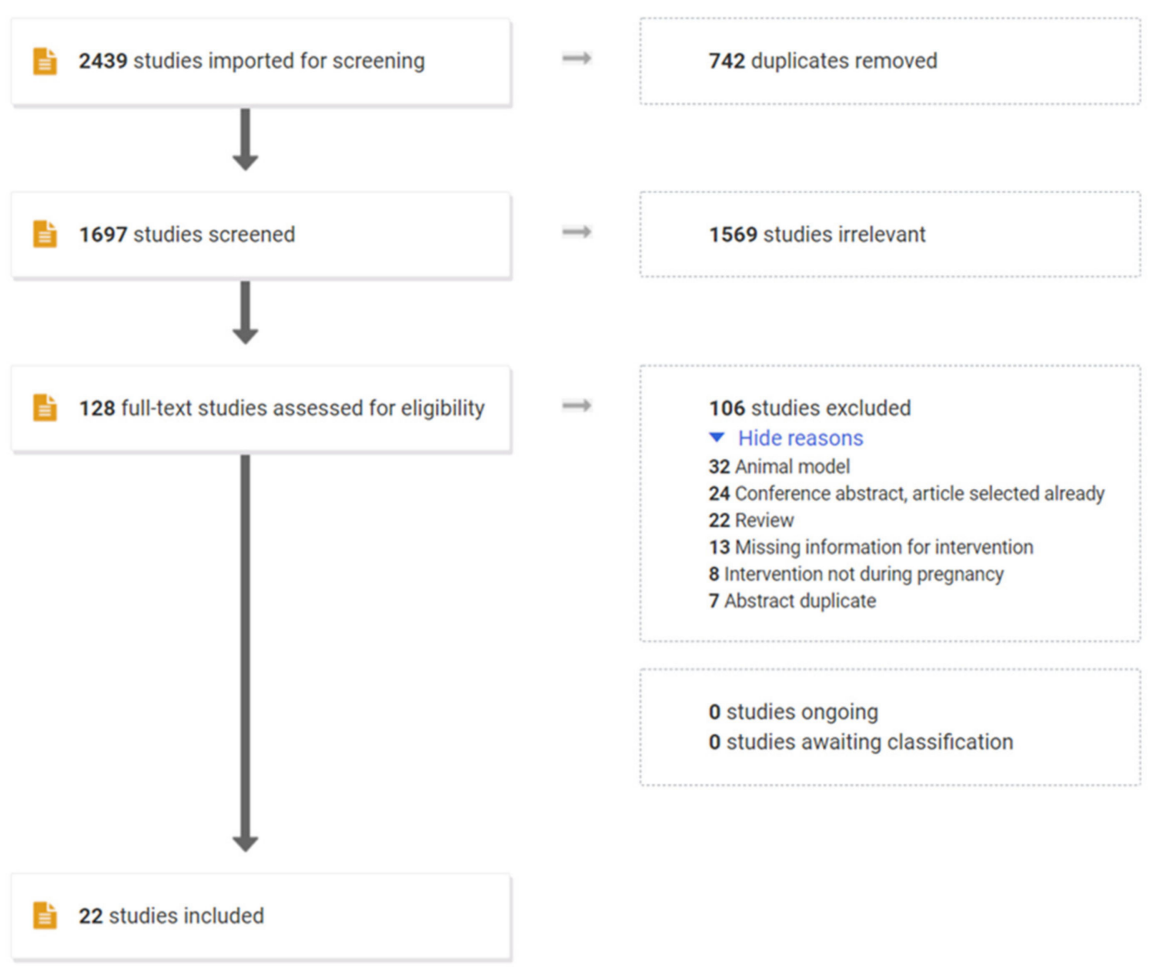

Figure 1. PRISMA flow chart of the systemic review of studies investigating the effect of IL-1 blockage during pregnancy.

\subsection{Risk of Bias/Methodological Quality of Included Studies}

The majority of studies included in this systematic review had a low risk of bias in the assessed domains as evaluated with the ROBINS-I tool. The majority of the case reports included adequate case ascertainment and follow-up, but there was limited data about the causal relationship between exposure to Anakinra and Canakinumab and adverse reactions (Table S1). It is important to note that almost half of the studies included in this systematic review were published conference abstracts and therefore provided limited data which could impact the results presented. Furthermore, five pregnancies were exposed to both Anakinra and Canakinumab which could affect the classification of the intervention and their outcomes. 


\subsection{Study Characteristics}

Characteristics of each study and summary of findings are presented in Table 1. Within the 22 studies included, 88 individual pregnancies were reported. 75 pregnancies $(85.2 \%)$ received Anakinra and $13(14.8 \%)$ Canakinumab. Of these 88 pregnancies, 5.7\% were exposed to both agents over the course of their pregnancy. The indications for these treatments were mostly cryopyrin-associated periodic syndrome-CAPS (34.1\%), including familial cold autoinflammatory syndrome-FCAS, neonatal-onset multisystem inflammatory disorder-NOMID and Muckle-Wells syndrome-MWS; familial Mediterranean fever-FMF (33.0\%); and adult-onset Still's disease-AOSD or systemic juvenile idiopathic arthritis-SJIA (20.4\%). The remaining cases (11 women/12.5\%) received treatment for the "TNF receptor associated periodic syndrome"-TRAPS (3.4\%), haemophagocytic lymphohistiocytosis-HLH (2.3\%) or other pathologies such as idiopathic pericarditis, Cogan syndrome or chronic inflammatory rheumatic disease $(6.8 \%)$.

Table 1. Characteristics and summary from studies included.

\begin{tabular}{|c|c|c|c|c|c|c|c|c|}
\hline Study & Study Design & $\begin{array}{c}\text { Year of } \\
\text { Publication }\end{array}$ & Population & $\begin{array}{l}\text { Number of } \\
\text { Pregnancies } \\
\text { Included in } \\
\text { This Study }\end{array}$ & $\begin{array}{l}\text { Indication for } \\
\text { Treatment }\end{array}$ & Treatment; Doses & Outcome & Notes \\
\hline 1 & $\begin{array}{l}\text { Case } \\
\text { report }\end{array}$ & 2009 & 1 & 1 & AOSD & $\begin{array}{c}\text { Anakinra; } \\
100 \text { mg/day }\end{array}$ & Healthy term baby & \\
\hline 2 & $\begin{array}{l}\text { Case } \\
\text { report }\end{array}$ & 2011 & 2 & 2 & AOSD & $\begin{array}{c}\text { Anakinra; } \\
100 \text { mg/day }\end{array}$ & $\begin{array}{c}\text { Two healthy babies, one } \\
\text { PTB } 36 \text { weeks }\end{array}$ & \\
\hline 3 & $\begin{array}{l}\text { Retrospective } \\
\text { cohort study }\end{array}$ & 2013 & 51 & 1 & SOJIA & Anakinra; NA & Term baby & $\begin{array}{l}\text { Big cohort of } \\
\text { SOJIA patients } \\
\text { but only one } \\
\text { pregnant }\end{array}$ \\
\hline 4 & $\begin{array}{l}\text { Prospective } \\
\text { cohort study }\end{array}$ & 2014 & 9 & 9 & $\begin{array}{c}\text { FCAS (6) } \\
\text { NOMID (1) } \\
\text { MWS/NOMID } \\
\text { (1) }\end{array}$ & $\begin{array}{c}\text { Anakinra; mostly } \\
100 \mathrm{mg} / \text { day but } \\
\text { also } \\
239-300 \mathrm{mg} / \text { day }\end{array}$ & $\begin{array}{l}\text { All term babies, three } \\
\text { with FCAS, one with } \\
\text { MWS and one twin } \\
\text { pregnancy resulted in } \\
\text { one death at } 30 \text { weeks }\end{array}$ & \\
\hline 5 & $\begin{array}{l}\text { Prospective } \\
\text { cohort study }\end{array}$ & 2015 & 4 & 0 & FMF & $\begin{array}{c}\text { Anakinra; } \\
100 \text { mg/day }\end{array}$ & $\begin{array}{c}\text { All healthy babies, one } \\
\text { PTB } 36 \text { weeks }\end{array}$ & $\begin{array}{c}\text { Data in } \\
\text { another study } \\
\text { already } \\
\text { included }\end{array}$ \\
\hline 6 & $\begin{array}{l}\text { Prospective } \\
\text { cohort study }\end{array}$ & 2015 & 6 & 3 & FMF & $\begin{array}{c}\text { Anakinra; } \\
100 \text { mg/day or NA }\end{array}$ & $\begin{array}{l}\text { All healthy babies, one } \\
\text { PTB } 36 \text { weeks }\end{array}$ & $\begin{array}{c}\text { Data in } \\
\text { another study } \\
\text { already } \\
\text { included }\end{array}$ \\
\hline 7 & $\begin{array}{l}\text { Prospective } \\
\text { cohort study }\end{array}$ & 2015 & 79 & 1 & $\begin{array}{l}\text { Chronic } \\
\text { inflammatory } \\
\text { rheumatic } \\
\text { disease }\end{array}$ & Anakinra; NA & $\begin{array}{l}\text { Voluntary pregnancy } \\
\text { termination }\end{array}$ & $\begin{array}{l}\text { Big cohort of } \\
\text { biological } \\
\text { drug } \\
\text { during } \\
\text { pregnancy, } \\
\text { only one took } \\
\text { anakinra }\end{array}$ \\
\hline 8 & $\begin{array}{l}\text { Case } \\
\text { report }\end{array}$ & 2017 & 1 & 1 & FMF & $\begin{array}{c}\text { Anakinra; } \\
100 \text { mg/day }\end{array}$ & Healthy term baby & \\
\hline 9 & $\begin{array}{l}\text { Prospective } \\
\text { cohort study }\end{array}$ & 2018 & 5 & 5 & $\begin{array}{l}\text { AOSD (3) } \\
\text { SOJIA (2) }\end{array}$ & $\begin{array}{c}\text { Anakinra; } \\
100 \text { mg/day }\end{array}$ & $\begin{array}{l}\text { All healthy term } \\
\text { babies but one with } \\
\text { right hydrocele, heart } \\
\text { murmur and resolved } \\
\text { low birthweight }\end{array}$ & \\
\hline 10 & $\begin{array}{l}\text { Retrospective } \\
\text { cohort study }\end{array}$ & 2018 & 4 & 4 & FMF & $\begin{array}{l}\text { Anakinra; } \\
100 \text { mg/day- } \\
2 \text { days }\end{array}$ & $\begin{array}{l}\text { All healthy babies but } \\
\text { one PTB at } 33 \text { weeks } \\
\text { with hypotrophic, } \\
\text { respiratory distress } \\
\text { syndrome, } \\
\text { hyperbilirubinemia and } \\
\text { poor drinking }\end{array}$ & \\
\hline
\end{tabular}


Table 1. Cont.

\begin{tabular}{|c|c|c|c|c|c|c|c|c|}
\hline Study & Study Design & $\begin{array}{c}\text { Year of } \\
\text { Publication }\end{array}$ & Population & $\begin{array}{l}\text { Number of } \\
\text { Pregnancies } \\
\text { Included in } \\
\text { This Study }\end{array}$ & $\begin{array}{l}\text { Indication for } \\
\text { Treatment }\end{array}$ & Treatment; Doses & Outcome & Notes \\
\hline 11 & $\begin{array}{l}\text { Prospective } \\
\text { cohort study }\end{array}$ & 2019 & 13 & 12 & FMF & $\begin{array}{c}\text { Anakinra; } \\
100 \text { mg/day or NA }\end{array}$ & $\begin{array}{l}\text { One miscarriage, two } \\
\text { PTB, one stillbirth but } \\
\text { overall healthy babies }\end{array}$ & $\begin{array}{c}\text { Two } \\
\text { pregnancies } \\
\text { still ongoing, } \\
\text { no } \\
\text { obstetrical } \\
\text { information } \\
\text { and two } \\
\text { pregnancies } \\
\text { with data in } \\
\text { another study } \\
\text { already } \\
\text { included }\end{array}$ \\
\hline 12 & $\begin{array}{l}\text { Case } \\
\text { report }\end{array}$ & 2019 & 1 & 1 & FMF & $\begin{array}{c}\text { Anakinra; } \\
100 \text { mg/day }\end{array}$ & Term healthy baby & $\begin{array}{c}\text { Cohort of four } \\
\text { patients with } \\
\text { FMF, only one } \\
\text { pregnant }\end{array}$ \\
\hline 13 & $\begin{array}{l}\text { Prospective } \\
\text { cohort study }\end{array}$ & 2019 & 54 & 1 & FMF & $\begin{array}{c}\text { Anakinra; } \\
100 \text { mg/day }\end{array}$ & $\begin{array}{c}\text { Obstetrical and } \\
\text { neonatal information } \\
\text { NA }\end{array}$ & $\begin{array}{l}\text { Cohort of } \\
\text { patient with } \\
\text { FMF, only one } \\
\text { pregnant }\end{array}$ \\
\hline 14 & $\begin{array}{l}\text { Case } \\
\text { report }\end{array}$ & 2019 & 1 & 1 & HLH & $\begin{array}{c}\text { Anakinra; } \\
200 \text { mg/twice daily }\end{array}$ & $\begin{array}{c}\text { Healthy but had } \\
\text { anaemia and marrow } \\
\text { suppression }\end{array}$ & \\
\hline 15 & $\begin{array}{l}\text { Retrospective } \\
\text { cohort study }\end{array}$ & 2020 & 16 & 3 & AOSD & Anakinra; NA & $\begin{array}{c}\text { All healthy babies but } \\
\text { one had PTB at } \\
28 \text { weeks }\end{array}$ & $\begin{array}{l}\text { Cohort of } \\
\text { child } \\
\text { exposed to } \\
\text { DMARDs, } \\
\text { only 3 } \\
\text { exposed to } \\
\text { anakinra } \\
\text { during } \\
\text { pregnancy }\end{array}$ \\
\hline 16 & $\begin{array}{l}\text { Case } \\
\text { report }\end{array}$ & 2020 & 1 & 1 & HLH & Anakinra; NA & $\begin{array}{c}\text { PTB at } 31 \text { weeks and } \\
\text { IUGR but overall } \\
\text { healthy }\end{array}$ & \\
\hline 17 & $\begin{array}{l}\text { Case } \\
\text { report }\end{array}$ & 2017 & 1 & 1 & MWS & $\begin{array}{l}\text { Canakinumab; } \\
150 \mathrm{mg} / \\
4-8 \text { weeks }\end{array}$ & Healthy term baby & \\
\hline 18 & $\begin{array}{l}\text { Case } \\
\text { report }\end{array}$ & 2018 & 1 & 1 & SOJIA & Canakinumab; NA & Healthy term baby & \\
\hline 19 & $\begin{array}{l}\text { Retrospective } \\
\text { cohort study }\end{array}$ & 2020 & 23 & 1 & FMF & $\begin{array}{l}\text { Canakinumab; } \\
150 \mathrm{mg} / \\
\text { 6-8 weeks }\end{array}$ & $\begin{array}{l}\text { One healthy term } \\
\text { pregnancy and one } \\
\text { without information }\end{array}$ & $\begin{array}{l}\text { Cohort of } \\
\text { patient with } \\
\text { FMF, only } \\
2 \text { pregnant }\end{array}$ \\
\hline 20 & $\begin{array}{l}\text { Retrospective } \\
\text { cohort study }\end{array}$ & 2013 & 7 & 7 & $\begin{array}{c}\text { AOSD (1) } \\
\text { CAPS (3) } \\
\text { TRAPS (1) } \\
\text { FMF (1) } \\
\text { Idiopathic } \\
\text { pericarditis (1) }\end{array}$ & $\begin{array}{c}\text { Anakinra; NA (6), } \\
\text { Canakinumab; } \\
\text { NA (1) }\end{array}$ & $\begin{array}{l}\text { All healthy babies, one } \\
\text { PTB } 36 \text { weeks and one } \\
\text { with unilateral reduced } \\
\text { hearing at } 6 \text { weeks }\end{array}$ & $\begin{array}{c}\text { Two } \\
\text { pregnancies } \\
\text { still ongoing, } \\
\text { no } \\
\text { obstetrical } \\
\text { information }\end{array}$ \\
\hline 21 & $\begin{array}{l}\text { Case } \\
\text { report }\end{array}$ & 2015 & 1 & 1 & MWS & $\begin{array}{l}\text { Canakinumab; NA } \\
\text { and Anakinra; NA }\end{array}$ & Healthy but with CAPS & \\
\hline 22 & $\begin{array}{l}\text { Retrospective } \\
\text { cohort study }\end{array}$ & 2017 & 43 & 31 & $\begin{array}{c}\text { AOSD (4) } \\
\text { CAPS (16) } \\
\text { Cogan } \\
\text { syndrome (2) } \\
\text { FMF (5) } \\
\text { Idiopathic } \\
\text { pericarditis (1) } \\
\text { TRAPS (2) } \\
\text { Un-SAID (1) }\end{array}$ & $\begin{array}{l}\text { Anakinra; mostly } \\
100 \mathrm{mg} / \text { day but } \\
\text { also } \\
\text { 50-300 mg/day } \\
\text { Canakinumab; } \\
150 \mathrm{mg} / \\
4-8 \text { weeks }\end{array}$ & $\begin{array}{l}\text { Two miscarriage (same } \\
\text { women), two PTB, all } \\
\text { healthy } \\
\text { babies but one with left } \\
\text { renal agenesis and } \\
\text { ectopic } \\
\text { neurohypophysis with } \\
\text { hormone } \\
\text { deficiency }\end{array}$ & $\begin{array}{l}43 \text { pregnancies } \\
\text { exposed to } \\
\text { IL-1 } \\
\text { inhibitor but } \\
11 \text { were male } \\
\text { exposure }\end{array}$ \\
\hline
\end{tabular}

HLH: hemophagocytic lymphohistiocytosis, FMF: familial mediterranean fever, AOSD: adult-onset Still's disease, FCAS: familial cold autoinflammatory Syndrome, NOMID: neonatal-onset multisystem inflammatory disease, MWS: Muckle-Wells syndrome, sJIA: systemic juvenile idiopathic arthritis, CAPS: cryopyrin-associated autoinflammatory syndromes, NA: not available.

Of the 88 pregnancies, 4 women $(4.5 \%)$ were still pregnant at the time of publication without any follow up available for their pregnancies. Of these women, three were within 
their first trimester and one in the second trimester, all without any complication reported to date. In the rest of pregnancies, three (3.4\%) resulted in miscarriage during the first trimester (two exposed to Anakinra and one to Canakinumab). Two of these spontaneous miscarriages occurred in the same patient, the first whilst on Canakinumab and the second with Anakinra since she presented with refractory Cogan syndrome. Unfortunately, the patient only had a partial clinical and biochemical response of her underlying diseases despite dose escalation of both treatment regimens. Finally, one patient on Anakinra terminated her pregnancy electively. For the rest of the analysis, these patients were excluded due to the lack of information.

\subsection{Duration of Exposure to Drugs during Pregnancy}

In 48 cases $(60.0 \%)$ of the remaining 80 pregnancies, the women were already taking the medication prior to getting pregnant; in 6 cases, this was unknown. In 50 cases $(62.5 \%)$, the drug therapy was continued throughout pregnancy (when it was started either prior to or during the first trimester until birth). In nine cases, treatment was stopped after the first trimester and, in 2 cases, after the second trimester, due to the lack of data on safety of these drugs in pregnancy. In 13 cases, treatment was started either during the second half of pregnancy (10 patients) or during the third trimester ( 3 patients). Of these 13 cases, 4 were due to a lack of improvement with their previous treatment (i.e., colchicine or prednisone), 2 women were diagnosed with AOSD or HLH while pregnant and no information was given for the remaining seven women. Details of the treatment duration is shown in a flow chart (Figure 2). Information concerning each pregnancy separately are shown in Table 2.

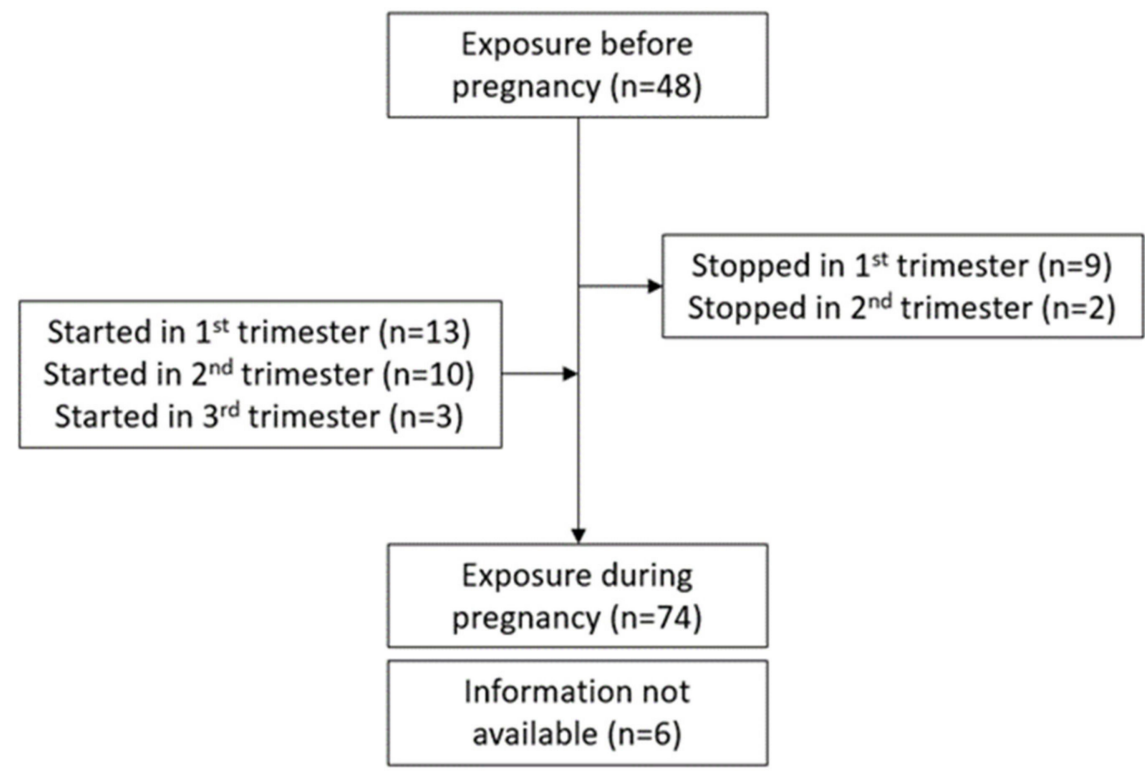

Figure 2. Flow chart of IL-1 blockade exposure during pregnancy (for the 11 pregnancies that stopped treatment during their 1st or 2nd trimester, treatment was initiated before conception and therefore they were included in the $n=48$ ). 
Table 2. Pregnancies details with maternal characteristic and neonatal outcomes.

\begin{tabular}{|c|c|c|c|c|c|c|c|c|c|c|c|c|}
\hline $\begin{array}{l}\text { Pregnancy } \\
\text { ID }\end{array}$ & $\begin{array}{l}\text { Pregnancy } \\
\text { from Study }\end{array}$ & $\begin{array}{c}\text { Indication } \\
\text { for } \\
\text { Treatment }\end{array}$ & Treatment & Doses & $\begin{array}{l}\text { Exposure } \\
\text { Time }\end{array}$ & $\begin{array}{l}\text { Mode of } \\
\text { Delivery }\end{array}$ & $\begin{array}{c}\text { GA at } \\
\text { Delivery } \\
\text { (Weeks) }\end{array}$ & $\begin{array}{c}\text { Birth Weight } \\
\text { (g) }\end{array}$ & $\begin{array}{l}\text { Obstetric } \\
\text { Complica- } \\
\text { tion }\end{array}$ & Child Sex & $\begin{array}{c}\text { Child } \\
\text { Wellbeing }\end{array}$ & Breasfeeding \\
\hline 1 & 14 & HLH & Anakinra & $\begin{array}{l}200 \mathrm{mg} / \\
\text { twice daily }\end{array}$ & $22 \mathrm{w}-\mathrm{B}$ & $\begin{array}{c}\text { C- } \\
\text { section }\end{array}$ & NA & NA & None & NA & $\begin{array}{c}\text { Anaemia and } \\
\text { bone marrow } \\
\text { suppression }\end{array}$ & NA \\
\hline 2 & 19 & $\begin{array}{c}\text { FMF + } \\
\text { amyloidosis }\end{array}$ & Canakinumab & $\begin{array}{c}150 \mathrm{mg} / 6 \\
\text { weeks }\end{array}$ & PC-8 w & NA & Term & NA & None & NA & Healthy & NA \\
\hline 3 & 19 & FMF & Canakinumab & $\begin{array}{c}150 \mathrm{mg} / 8 \\
\text { weeks }\end{array}$ & PC-PPT & NA & NA & NA & NA & NA & NA & NA \\
\hline $4^{*}$ & 7 & $\begin{array}{l}\text { Chronic in- } \\
\text { flammatory } \\
\text { rheumatic } \\
\text { disease }\end{array}$ & Anakinra & NA & NA & Vaginal & NA & NA & $\begin{array}{l}\text { Voluntary } \\
\text { abortion }\end{array}$ & NA & NA & NA \\
\hline 5 & 1 & AOSD & Anakinra & $\begin{array}{c}100 \mathrm{mg} / \\
\text { day }\end{array}$ & PC-B & Vaginal & 40.7 & 2700 & $\begin{array}{l}\text { Placental } \\
\text { retention } \\
\text { requiring } \\
\text { manual } \\
\text { abruption }\end{array}$ & $\mathrm{F}$ & Healthy & Yes \\
\hline 6 & 4 & FCAS & Anakinra & $\begin{array}{c}100 \mathrm{mg} / \\
\text { day }\end{array}$ & PC-B & Vaginal & 41.0 & 3742 & None & NA & Healthy & No \\
\hline 7 & 4 & FCAS & Anakinra & $\begin{array}{c}100 \mathrm{mg} / \\
\text { day }\end{array}$ & PC-B & Vaginal & 41.0 & 3629 & None & NA & FCAS & No \\
\hline 8 & 4 & FCAS & Anakinra & $\begin{array}{c}100 \mathrm{mg} / \\
\text { day }\end{array}$ & PC-B & Vaginal & 38.0 & 3402 & None & NA & FCAS & Yes \\
\hline 9 & 4 & FCAS & Anakinra & $\begin{array}{c}100 \mathrm{mg} / \\
\text { day }\end{array}$ & PC-B & Vaginal & 37.0 & 3459 & None & NA & Healthy & No \\
\hline 10 & 4 & FCAS & Anakinra & $\begin{array}{c}100 \mathrm{mg} / \\
\text { day }\end{array}$ & PC-B & Vaginal & 37.7 & 2977 & None & NA & FCAS & No \\
\hline 11 & 4 & FCAS & Anakinra & $\begin{array}{c}100 \mathrm{mg} / \\
\text { day }\end{array}$ & PC-B & Vaginal & 39.0 & 3345 & None & NA & Healthy & No \\
\hline 12 & 4 & NOMID & Anakinra & $\begin{array}{c}300 \mathrm{mg} / \\
\text { day }\end{array}$ & PC-B & $\begin{array}{c}\text { C- } \\
\text { section }\end{array}$ & 40.0 & 4139 & $\begin{array}{c}\text { Chronic } \\
\text { hypertension }\end{array}$ & NA & Healthy & Yes \\
\hline 13 & 4 & NOMID & Anakinra & $\begin{array}{l}\text { 239-300 mg/ } \\
\text { day }\end{array}$ & PC-B & Vaginal & $\begin{array}{l}\text { A: } 38.7 \\
\text { B: } 30.0\end{array}$ & $\begin{array}{l}\text { A: } 2637 \\
\text { B: NA }\end{array}$ & $\begin{array}{l}\text { A: None } \\
\text { B: PTB }\end{array}$ & NA & $\begin{array}{c}\text { A: Healthy B: } \\
\text { Renal } \\
\text { agenesis } \\
\text { (death) }\end{array}$ & $\begin{array}{l}\text { A: Yes } \\
\text { B: No }\end{array}$ \\
\hline
\end{tabular}


Table 2. Cont.

\begin{tabular}{|c|c|c|c|c|c|c|c|c|c|c|c|c|}
\hline $\begin{array}{l}\text { Pregnancy } \\
\text { ID }\end{array}$ & $\begin{array}{l}\text { Pregnancy } \\
\text { from Study }\end{array}$ & $\begin{array}{l}\text { Indication } \\
\quad \text { for } \\
\text { Treatment }\end{array}$ & Treatment & Doses & $\begin{array}{l}\text { Exposure } \\
\text { Time }\end{array}$ & $\begin{array}{l}\text { Mode of } \\
\text { Delivery }\end{array}$ & $\begin{array}{c}\text { GA at } \\
\text { Delivery } \\
\text { (Weeks) }\end{array}$ & $\begin{array}{c}\text { Birth Weight } \\
\text { (g) }\end{array}$ & $\begin{array}{l}\text { Obstetric } \\
\text { Complica- } \\
\text { tion }\end{array}$ & Child Sex & $\begin{array}{c}\text { Child } \\
\text { Wellbeing }\end{array}$ & Breasfeeding \\
\hline 14 & 4 & MWS/NOMID & Anakinra & $\begin{array}{c}100 \mathrm{mg} / \\
\text { day }\end{array}$ & PC-B & $\begin{array}{c}\mathrm{C}- \\
\text { section }\end{array}$ & Term & 3515 & None & NA & MWS & No \\
\hline 15 & 15 & AOSD & Anakinra & NA & PC-B & $\begin{array}{c}\mathrm{C}- \\
\text { section }\end{array}$ & 28.0 & 1175 & РТВ & $\mathrm{F}$ & Healthy & NA \\
\hline 16 & 15 & AOSD & Anakinra & NA & PC-B & Vaginal & 40.0 & 3480 & None & $\mathrm{M}$ & Healthy & $\mathrm{NA}$ \\
\hline 17 & 15 & AOSD & Anakinra & NA & PC-B & Vaginal & 38.0 & 3450 & None & $\mathrm{M}$ & Healthy & NA \\
\hline 20 & 17 & MWS & Canakinumab & $\begin{array}{l}150 \mathrm{mg} / \\
8 \text { weeks, } \\
\text { then every } \\
4-5 \text { weeks }\end{array}$ & PC $-34 \mathrm{w}$ & $\begin{array}{c}\text { C- } \\
\text { section }\end{array}$ & 39.0 & 2994 & None & $\mathrm{F}$ & $\begin{array}{c}\text { Healthy with } \\
\text { NLRP3 } \\
\text { mutation }\end{array}$ & NA \\
\hline 22 & 11 & FMF & Anakinra & $\begin{array}{l}100 \mathrm{mg} / \\
\text { day }\end{array}$ & $\begin{array}{c}P C-29 w+33 \\
w-B\end{array}$ & $\begin{array}{c}\text { C- } \\
\text { section }\end{array}$ & 38.0 & NA & $\begin{array}{l}\text { Incision site } \\
\text { infection in } \\
\text { postpartum }\end{array}$ & $\mathrm{M}$ & Healthy & NA \\
\hline 24 & 11 & FMF & Anakinra & NA & $16 \mathrm{w}-\mathrm{B}$ & $\begin{array}{c}\mathrm{C}- \\
\text { section }\end{array}$ & 31.0 & NA & РТВ & F-F twins & Healthy & NA \\
\hline 25 & 11 & FMF & Anakinra & NA & $23 w-B$ & $\begin{array}{c}\text { C- } \\
\text { section }\end{array}$ & 37.0 & NA & NA & $\mathrm{F}$ & Healthy & NA \\
\hline 26 & 11 & FMF & Anakinra & NA & $32 \mathrm{w}-\mathrm{B}$ & $\begin{array}{c}\mathrm{C}- \\
\text { section }\end{array}$ & 40.0 & NA & NA & $\mathrm{F}$ & Healthy & NA \\
\hline 27 & 11 & FMF & Anakinra & NA & $\begin{array}{l}\text { PC-B with 1 } \\
\text { month } \\
\text { interruption }\end{array}$ & $\begin{array}{c}\text { C- } \\
\text { section }\end{array}$ & 38.0 & NA & NA & $\mathrm{F}$ & Healthy & NA \\
\hline 28 & 11 & FMF & Anakinra & NA & $34 \mathrm{w}-\mathrm{B}$ & Vaginal & 37.0 & $\mathrm{NA}$ & Stillbirth & $\mathrm{M}$ & & $\mathrm{NA}$ \\
\hline 29 & 11 & FMF & Anakinra & NA & $6 \mathrm{w}-\mathrm{B}$ & $\begin{array}{c}\text { C- } \\
\text { section }\end{array}$ & 36.0 & NA & РТВ & $\mathrm{F}$ & Healthy & NA \\
\hline 30 & 11 & FMF & Anakinra & NA & NA & $\mathrm{NA}$ & NA & NA & NA & NA & NA & NA \\
\hline 31 * & 11 & FMF & Anakinra & NA & $\begin{array}{l}5 w-8 w \\
\text { (ongoing) }\end{array}$ & NA & NA & NA & $\mathrm{NA}$ & NA & NA & NA \\
\hline $32 *$ & 11 & FMF & Anakinra & NA & $\begin{array}{l}\text { PC-8 w } \\
\text { (ongoing) }\end{array}$ & $\mathrm{NA}$ & NA & NA & NA & NA & NA & NA \\
\hline
\end{tabular}


Table 2. Cont.

\begin{tabular}{|c|c|c|c|c|c|c|c|c|c|c|c|c|}
\hline $\begin{array}{l}\text { Pregnancy } \\
\text { ID }\end{array}$ & $\begin{array}{l}\text { Pregnancy } \\
\text { from Study }\end{array}$ & $\begin{array}{c}\text { Indication } \\
\text { for } \\
\text { Treatment }\end{array}$ & Treatment & Doses & $\begin{array}{l}\text { Exposure } \\
\text { Time }\end{array}$ & $\begin{array}{l}\text { Mode of } \\
\text { Delivery }\end{array}$ & $\begin{array}{c}\text { GA at } \\
\text { Delivery } \\
\text { (Weeks) }\end{array}$ & $\begin{array}{c}\text { Birth Weight } \\
\text { (g) }\end{array}$ & $\begin{array}{l}\text { Obstetric } \\
\text { Complica- } \\
\text { tion }\end{array}$ & Child Sex & $\begin{array}{c}\text { Child } \\
\text { Wellbeing }\end{array}$ & Breasfeeding \\
\hline 33 & 2 & AOSD & Anakinra & $\begin{array}{c}100 \mathrm{mg} / \\
\text { day }\end{array}$ & PC-B & Vaginal & 39.0 & 3100 & None & M & Healthy & No \\
\hline 34 & 2 & AOSD & Anakinra & NA & $12 \mathrm{w}-\mathrm{B}$ & $\begin{array}{c}\mathrm{C}- \\
\text { section }\end{array}$ & 36.0 & 2800 & РTB & $\mathrm{M}$ & Healthy & No \\
\hline 35 & 18 & sJIA & Canakinumab & NA & PC-35 w & Vaginal & 39.0 & NA & $\begin{array}{c}\text { Forceps + } \\
\text { minor } \\
\text { episiotomy } \\
\text { wound } \\
\text { infection }\end{array}$ & $\mathrm{M}$ & Healthy & NA \\
\hline 36 & 21 & MWS & $\begin{array}{c}\text { Canakinumab } \\
\text { and Ana } \\
\text { kinra }\end{array}$ & NA & PC-B & NA & NA & NA & NA & $\mathrm{M}$ & $\begin{array}{c}\text { Healthy with } \\
\text { CAPS }\end{array}$ & Yes \\
\hline 37 & 8 & FMF & Anakinra & $\begin{array}{c}100 \mathrm{mg} / \\
\text { day }\end{array}$ & PC-B & $\begin{array}{c}\text { C- } \\
\text { section }\end{array}$ & 38.0 & 2700 & None & NA & Healthy & Yes \\
\hline $38 *$ & 20 & CAPS & Anakinra & NA & $\begin{array}{c}\text { PC-NA } \\
\text { (ongoing) }\end{array}$ & NA & NA & NA & NA & NA & NA & NA \\
\hline 40 & 20 & CAPS & Anakinra & NA & PC-B & Vaginal & NA & NA & None & $\mathrm{M}$ & Healthy & No \\
\hline 41 & 20 & TRAPS & Anakinra & NA & PC-B & Vaginal & NA & NA & None & $\mathrm{M}$ & $\begin{array}{c}\text { Unilateral } \\
\text { reduced } \\
\text { hearing at } 6 \\
\text { weeks }\end{array}$ & No \\
\hline 42 & 20 & FMF & Anakinra & $\begin{array}{c}100 \mathrm{mg} / \\
\text { day }\end{array}$ & $21 \mathrm{w}-\mathrm{B}$ & $\begin{array}{c}\text { C- } \\
\text { section }\end{array}$ & 36.0 & NA & $\begin{array}{l}\text { Vaginal } \\
\text { bleeding, } \\
\text { PTB }\end{array}$ & $\mathrm{M}$ & Healthy & Yes \\
\hline 43 & 20 & $\begin{array}{l}\text { idiopathic } \\
\text { pericarditis }\end{array}$ & Anakinra & NA & PC-B & Vaginal & NA & NA & None & $\mathrm{M}$ & Healthy & No \\
\hline 44 & 20 & AOSD & Anakinra & NA & $22 w-33 w$ & Vaginal & NA & NA & None & $\mathrm{M}$ & Healthy & No \\
\hline 50 & 6 & FMF & Anakinra & $\begin{array}{c}100 \mathrm{mg} / \\
\text { day }\end{array}$ & $12 \mathrm{w}-\mathrm{B}$ & Vaginal & 40.0 & NA & None & $\mathrm{F}$ & Healthy & Yes \\
\hline
\end{tabular}


Table 2. Cont.

\begin{tabular}{|c|c|c|c|c|c|c|c|c|c|c|c|c|}
\hline $\begin{array}{l}\text { Pregnancy } \\
\text { ID }\end{array}$ & $\begin{array}{l}\text { Pregnancy } \\
\text { from Study }\end{array}$ & $\begin{array}{l}\text { Indication } \\
\text { for } \\
\text { Treatment }\end{array}$ & Treatment & Doses & $\begin{array}{l}\text { Exposure } \\
\text { Time }\end{array}$ & $\begin{array}{l}\text { Mode of } \\
\text { Delivery }\end{array}$ & $\begin{array}{l}\text { GA at } \\
\text { Delivery } \\
\text { (Weeks) }\end{array}$ & $\begin{array}{l}\text { Birth Weight } \\
\text { (g) }\end{array}$ & $\begin{array}{l}\text { Obstetric } \\
\text { Complica- } \\
\text { tion }\end{array}$ & Child Sex & $\begin{array}{c}\text { Child } \\
\text { Wellbeing }\end{array}$ & Breasfeeding \\
\hline 52 & 6 & FMF & Anakinra & NA & $15 \mathrm{w}-\mathrm{B}$ & Vaginal & 38.0 & NA & None & M & $\begin{array}{c}\text { Low } \\
\text { thrombocyte } \\
\text { count treated } \\
\text { by IVIG }\end{array}$ & NA \\
\hline 54 & 3 & sJIA & Anakinra & NA & $\mathrm{P}-\mathrm{B}$ & NA & Term & NA & NA & NA & NA & NA \\
\hline 55 & 13 & FMF & Anakinra & $\begin{array}{c}100 \mathrm{mg} / \\
\text { day }\end{array}$ & P-B & NA & NA & NA & None & NA & NA & NA \\
\hline 56 & 9 & sJIA & Anakinra & $\begin{array}{c}100 \mathrm{mg} / \\
\text { day }\end{array}$ & PC-20.4 w & $\begin{array}{c}\text { C- } \\
\text { section }\end{array}$ & 37.1 & 2419 & $\begin{array}{l}\text { Hypertension, } \\
\text { oligohydram- } \\
\text { nios, breech } \\
\text { presentation }\end{array}$ & M & $\begin{array}{l}\text { Jaundice, } \\
\text { right } \\
\text { hydrocele } \\
\text { and heart } \\
\text { murmur }\end{array}$ & No \\
\hline 57 & 9 & AOSD & Anakinra & $\begin{array}{c}100 \mathrm{mg} / \\
\text { day }\end{array}$ & $20 w-38.1 w$ & Vaginal & 40.1 & 2940 & None & M & Jaundice & NA \\
\hline 58 & 9 & AOSD & Anakinra & $\begin{array}{c}100 \mathrm{mg} / \\
\text { day }\end{array}$ & $\begin{array}{c}\text { PC- } \\
16.6 \mathrm{w}+19.4 \\
\mathrm{w}- \\
37.3 \mathrm{w}\end{array}$ & $\begin{array}{c}\text { C- } \\
\text { section }\end{array}$ & 39.4 & 3632 & None & M & Jaundice & Yes \\
\hline 59 & 9 & AOSD & Anakinra & $\begin{array}{c}100 \mathrm{mg} / \\
\text { day }\end{array}$ & $\begin{array}{c}\text { PC- } \\
2 \mathrm{w}+9.6 \mathrm{w}- \\
36.7 \mathrm{w}\end{array}$ & Vaginal & 38.7 & 3519 & None & M & Tongue-tied & Yes \\
\hline 60 & 9 & sJIA & Anakinra & $\begin{array}{c}100 \mathrm{mg} / \\
\text { day }\end{array}$ & $\begin{array}{c}\text { PC- } \\
37.3 \mathrm{w}\end{array}$ & Vaginal & 39.4 & 2640 & Oligohydramnios & $\mathrm{F}$ & Healthy & No \\
\hline 61 & 10 & FMF & Anakinra & $\begin{array}{c}100 \mathrm{mg} / \\
\text { day }\end{array}$ & $\mathrm{P}-\mathrm{B}$ & $\begin{array}{c}\text { C- } \\
\text { section }\end{array}$ & 40.6 & 4025 & None & NA & Healthy & Yes \\
\hline 62 & 10 & FMF & Anakinra & $\begin{array}{l}100 \mathrm{mg} / \\
\text { day }\end{array}$ & $\begin{array}{c}2 \mathrm{e} \\
\text { trimester-B }\end{array}$ & $\begin{array}{c}\text { C- } \\
\text { section }\end{array}$ & 33.7 & 3320 & PTB & NA & $\begin{array}{c}\text { Healthy, } \\
\text { hypotrophic, } \\
\text { respiratory } \\
\text { distress } \\
\text { syndrome, } \\
\text { hyperbiliru- } \\
\text { binemia and } \\
\text { poor } \\
\text { drinking }\end{array}$ & No \\
\hline 63 & 10 & FMF & Anakinra & $\begin{array}{c}100 \mathrm{mg} / 2 \\
\text { days }\end{array}$ & P-B & $\begin{array}{c}\text { C- } \\
\text { section }\end{array}$ & 39.3 & 4030 & $\begin{array}{l}\text { Premature } \\
\text { bleeding }\end{array}$ & NA & Healthy & NA \\
\hline
\end{tabular}


Table 2. Cont.

\begin{tabular}{|c|c|c|c|c|c|c|c|c|c|c|c|c|}
\hline $\begin{array}{l}\text { Pregnancy } \\
\text { ID }\end{array}$ & $\begin{array}{l}\text { Pregnancy } \\
\text { from Study }\end{array}$ & $\begin{array}{c}\text { Indication } \\
\text { for } \\
\text { Treatment }\end{array}$ & Treatment & Doses & $\begin{array}{c}\text { Exposure } \\
\text { Time }\end{array}$ & $\begin{array}{l}\text { Mode of } \\
\text { Delivery }\end{array}$ & $\begin{array}{c}\text { GA at } \\
\text { Delivery } \\
\text { (Weeks) }\end{array}$ & $\begin{array}{l}\text { Birth Weight } \\
\text { (g) }\end{array}$ & $\begin{array}{l}\text { Obstetric } \\
\text { Complica- } \\
\text { tion }\end{array}$ & Child Sex & $\begin{array}{c}\text { Child } \\
\text { Wellbeing }\end{array}$ & Breasfeeding \\
\hline 64 & 10 & FMF & Anakinra & $\begin{array}{c}100 \mathrm{mg} / 2 \\
\text { days }\end{array}$ & $\mathrm{P}-\mathrm{B}$ & $\begin{array}{c}\text { C- } \\
\text { section }\end{array}$ & 36.4 & 3320 & РTB & NA & Healthy & NA \\
\hline 66 & 16 & HLH & Anakinra & NA & $22 \mathrm{w}-\mathrm{B}$ & $\begin{array}{c}\text { C- } \\
\text { section }\end{array}$ & 31.7 & NA & $\begin{array}{l}\text { PTB, IUGR, } \\
\text { abnormal } \\
\text { umbilical } \\
\text { artery } \\
\text { Doppler and } \\
\text { subsequent } \\
\text { cardiotocog- } \\
\text { raphy was } \\
\text { abnormal }\end{array}$ & $\mathrm{M}$ & $\begin{array}{l}\text { Neonatal } \\
\text { unit briefly } \\
\text { but healthy }\end{array}$ & NA \\
\hline 67 & 22 & CAPS & Canakinumab & $\begin{array}{l}150 \mathrm{mg} / \\
8 \text { weeks }\end{array}$ & PC-8 w & $\begin{array}{c}\mathrm{C}- \\
\text { section }\end{array}$ & 38.0 & 3540 & $\begin{array}{c}\text { Gestationnal } \\
\text { diabetes }\end{array}$ & $\mathrm{M}$ & Healthy & No \\
\hline 68 & 22 & CAPS & Canakinumab & $\begin{array}{l}150 \mathrm{mg} / \\
8 \text { weeks }\end{array}$ & PC $-12 \mathrm{w}$ & Vaginal & 40.0 & 4480 & None & $\mathrm{F}$ & Healthy & Yes \\
\hline 69 & 22 & CAPS & Canakinumab & $\begin{array}{l}150 \mathrm{mg} / \\
8 \text { weeks }\end{array}$ & $1 w-36 w$ & NA & 40.0 & 3570 & None & $\mathrm{M}$ & Healthy & NA \\
\hline 71 & 22 & Un-SAID & Canakinumab & $\begin{array}{l}300 \mathrm{mg} / \\
8 \text { weeks }\end{array}$ & PC-B & Vaginal & 39.0 & NA & None & M & Healthy & NA \\
\hline 72 & 22 & FMF & Canakinumab & $\begin{array}{l}150 \mathrm{mg} / \\
4 \text { weeks }\end{array}$ & PC-B & $\begin{array}{c}\text { C- } \\
\text { section }\end{array}$ & 37.0 & 3300 & None & $\mathrm{M}$ & Healthy & Yes \\
\hline 73 & 22 & FMF & Canakinumab & $\begin{array}{l}150 \mathrm{mg} / \\
8 \text { weeks }\end{array}$ & PC $-4 \mathrm{w}$ & $\begin{array}{c}\text { C- } \\
\text { section }\end{array}$ & 40.0 & 3300 & None & $\mathrm{F}$ & Healthy & Yes \\
\hline $74^{*}$ & 22 & $\begin{array}{l}\text { Cogan } \\
\text { syndrome }\end{array}$ & Canakinumab & $\begin{array}{l}150 \mathrm{mg} / \\
4 \text { weeks }\end{array}$ & PC-4 w & Vaginal & 4.0 & NA & Miscarriage & NA & & NA \\
\hline 75 & 22 & CAPS & Anakinra & $\begin{array}{c}50 \mathrm{mg} / \\
\text { day }\end{array}$ & PC-B & Vaginal & 39.0 & 3940 & None & M & Healthy & No \\
\hline 76 & 22 & CAPS & Anakinra & $\begin{array}{c}50 \mathrm{mg} / \\
\text { day }\end{array}$ & PC-B & Vaginal & 39.0 & NA & None & $\mathrm{F}$ & Healthy & No \\
\hline 77 & 22 & CAPS & Anakinra & $\begin{array}{c}100 \mathrm{mg} / \\
\text { day }\end{array}$ & PC-B & Vaginal & 41.1 & 3600 & None & $\mathrm{M}$ & Healthy & Yes \\
\hline
\end{tabular}


Table 2. Cont.

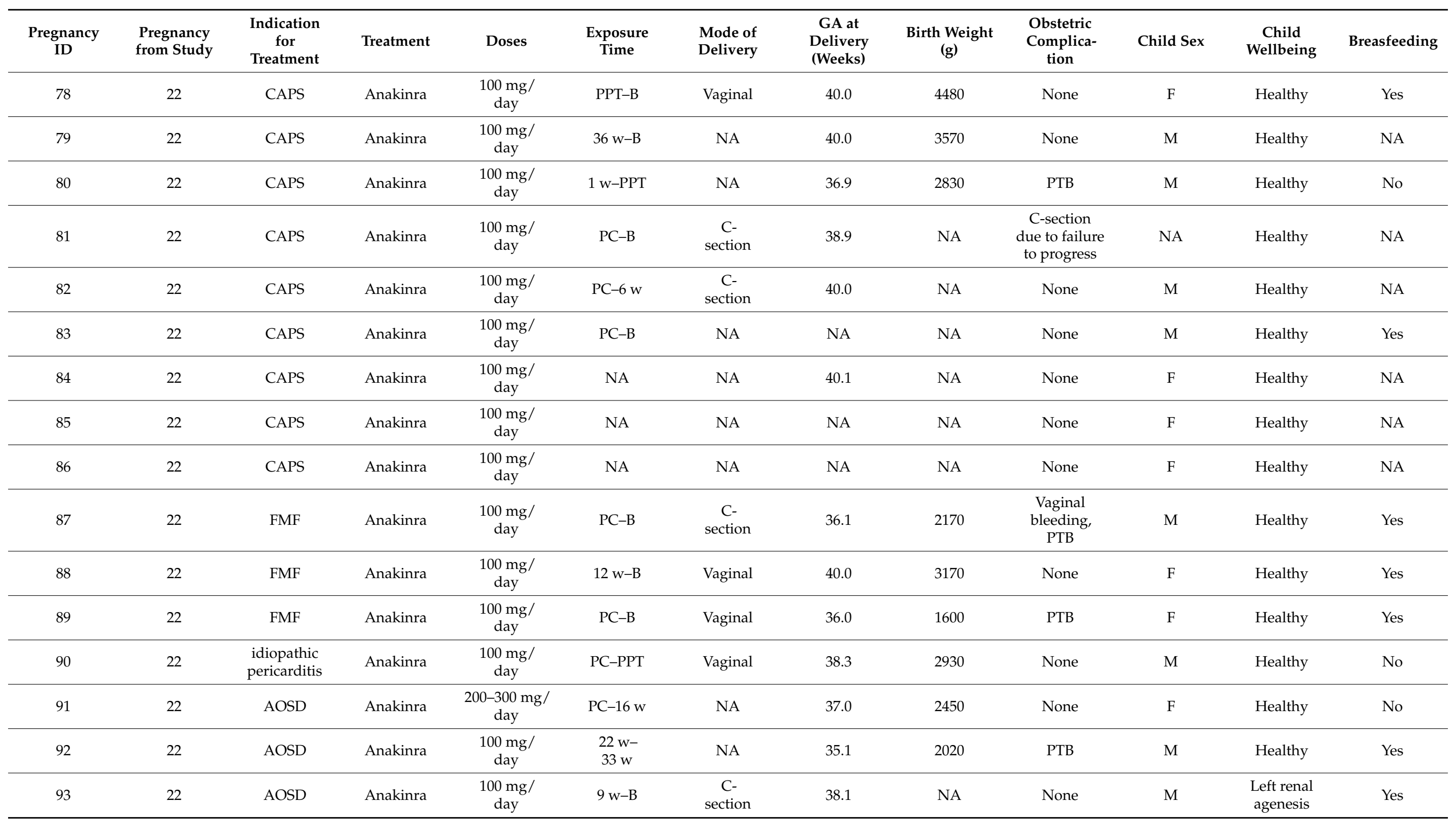


Table 2. Cont.

\begin{tabular}{|c|c|c|c|c|c|c|c|c|c|c|c|c|}
\hline $\begin{array}{l}\text { Pregnancy } \\
\text { ID }\end{array}$ & $\begin{array}{l}\text { Pregnancy } \\
\text { from Study }\end{array}$ & $\begin{array}{l}\text { Indication } \\
\text { for } \\
\text { Treatment }\end{array}$ & Treatment & Doses & $\begin{array}{l}\text { Exposure } \\
\text { Time }\end{array}$ & $\begin{array}{l}\text { Mode of } \\
\text { Delivery }\end{array}$ & $\begin{array}{c}\text { GA at } \\
\text { Delivery } \\
\text { (Weeks) }\end{array}$ & $\begin{array}{l}\text { Birth Weight } \\
\text { (g) }\end{array}$ & $\begin{array}{l}\text { Obstetric } \\
\text { Complica- } \\
\text { tion }\end{array}$ & Child Sex & $\begin{array}{c}\text { Child } \\
\text { Wellbeing }\end{array}$ & Breasfeeding \\
\hline 94 & 22 & AOSD & Anakinra & $\begin{array}{c}100 \mathrm{mg} / \\
\text { day }\end{array}$ & NA & Vaginal & 38.0 & 3060 & None & F & Healthy & Yes \\
\hline 95 & 22 & TRAPS & Anakinra & $\begin{array}{c}100 \mathrm{mg} / \\
\text { day }\end{array}$ & PC-B & Vaginal & 41.0 & 3230 & None & M & Healthy & Yes \\
\hline 96 & 22 & TRAPS & Anakinra & $\begin{array}{c}100 \mathrm{mg} / \\
\text { day }\end{array}$ & PC-B & NA & NA & NA & None & F & Healthy & NA \\
\hline
\end{tabular}

HLH: hemophagocytic lymphohistiocytosis, FMF: familial mediterranean fever, AOSD: adult-onset Still's disease, FCAS: familial cold autoinflammatory Syndrome, NOMID: neonatalonset multisystem inflammatory disease, MWS: Muckle-Wells syndrome, sJIA: systemic juvenile idiopathic arthritis, CAPS: cryopyrin-associated autoinflammatory syndromes, NA: not available, PC: prior to conception, B: birth, PPT: pregnancy positive test, P: pregnancy, GA: gestational age, PTB: preterm birth, IUGR: intra-uterine growth restriction, M: male, F: female, *: Excluded from further analysis since data missing. 


\subsection{Anakinra Use during Pregnancy and Maternal/Fetal Outcome}

Women treated with Anakinra received doses ranging from 50 to $200 \mathrm{mg} /$ daily, but the majority (59.4\%) received $100 \mathrm{mg} /$ day. In 20 cases $(29.0 \%)$, this information was unavailable.

Of the 69 pregnancies exposed to Anakinra, $63.8 \%$ had term births, $17.4 \%$ were preterm (mean gestational age: 34.1 weeks (range: $28-36.9)$ ) and for the rest (18.8\%) this information was not given. Overall, the mean gestational age at delivery in the Anakinra exposed group was 37.9 (28.0-41.1) weeks. Within Anakinra-exposed pregnancies, $63.8 \%$ had no adverse obstetric outcome and $26.1 \%$ had complications with the most predominant being preterm birth $(12 / 18)$ while the rest presented one or more of the following complication; vaginal bleeding, hypertension and/or oligohydramnios. One case ended in stillbirth, and the women received Anakinra for familial Mediterranean fever from 34 weeks of gestation to the time of stillbirth (37 weeks). No additional information was available about this event. It is also important to note that one twin dichorionic-diamniotic pregnancy occurred with the demise of one fetus due to bilateral renal agenesis at 30 weeks' gestation. However, the surviving twin had no abnormality and was born at 38.7 weeks. This pregnancy was treated from the first trimester with Anakinra for neonatal-onset multisystem inflammatory disorder. The obstetric data were not available in $10.1 \%$ of the cases.

As for the neonates, $86.4 \%$ were healthy whereas $13.6 \%$ presented some mild complications. Of these, five babies were diagnosed with CAPS whereas three presented with other problems such as hypotrophic, respiratory distress syndrome, renal agenesis, ectopic neurohypophysis, right hydrocele and/or heart murmur. Rates of breastfeeding were available for 42 pregnancies with half of them being breastfeed; however, it was not clear if the treatment was maintained during this time.

\subsection{Canakinumab Use during Pregnancy and Maternal/Fetal Outcome}

The women using Canakinumab received doses starting from a single $120 \mathrm{mg}$ dose to $300 \mathrm{mg} / 8$ weeks but $54.5 \%$ received $150 \mathrm{mg} / 8$ weeks. Of the 11 pregnancies exposed to Canakinumab, $90.9 \%$ delivered at term and the mean gestational age in this population was 38.8 (37.0-40.0) weeks whilst for the remaining one case the information was unavailable. In this group, $90.0 \%$ had no adverse obstetrical outcome with only one woman developing gestational diabetes and one without any information.

All the babies exposed to Canakinumab were healthy with one presenting the same NLPR3 mutation as the mother. Rates of breastfeeding were available for 5 cases and $80.0 \%$ were breastfeed but again, no data on treatment during this period.

\section{Discussion}

This systematic review aimed to review the effects of IL-1 antagonists used during pregnancy in humans. We found 22 studies including 12 original articles and 10 conference abstracts published which were reporting at least one pregnancy exposed to IL-1 blockade. Of these 22 studies, data extraction was performed, and 88 different pregnancies were included in this systematic review. Furthermore, some pregnancies were reported more than once and therefore the extraction were combined to obtained complete information whilst avoiding duplicates.

Of the 88 pregnancies included, $85.2 \%$ of women received Anakinra whereas 14.8\% received Canakinumab. This disparity could be due to the fact that Anakinra has been approved for therapeutic use for over 20 years as opposed to Canakinumab [65]. Furthermore, Canakinumab has a higher cost and is less widely used [77]. In a recent review by Soh and Moretto, the authors summarize the European League Against Rheumatism-EULAR and British Society on Rheumatology_BSR guidelines for biologic therapies used during pregnancy [61]. In the EULAR guidelines, Anakinra is tolerated in early pregnancy and can be continued during pregnancy if there are no other options. On the other hand, the BSR guidelines reports insufficient data to recommend the use of Anakinra during pregnancy, but stipulate that "unintentional use during first trimester is unlikely to cause 
harm". Furthermore, these guidelines states that it is "not recommended to continue the treatment during gestation". The data for Canakinumab are even more sparse. One case report measured its transplacental transfer and found a cord blood to maternal blood ratio of 2.11 [78] which needs to be further studied.

Autoimmune diseases often have negative impact on fertility and pregnancy outcomes [76,77]. Two factors can be considered to affect the course of pregnancy, the disease or the treatment for this disease. In the exposed pregnancies included in this review, the indications for treatment were CAPS (34.1\%), Familial Mediterranean Fever (FMF-33\%), and AOSD/SJIA (20.4\%). There is only one report, to our knowledge, of CAPS during pregnancy treated with medication other than those targeting the IL-1 system [78]. This study reported a rate of miscarriage of $30 \%$ as compared to $10 \%$ for CAPS-patients treated with Anakinra [78]. Only two studies reported pregnancy with those pathologies all exposed to Anakinra or Canakinumab, therefore it is difficult to distinguish the treatment effect to that from the inflammatory pathology itself $[75,78]$. There is only one report of untreated pregnancies with different autoimmune disease that the one that are reported in this study making the evaluation of the pathologies themselves difficult. In this study, the authors compared pregnancy with or without all kind of DMARDs and healthy pregnancy. However, this study did not discriminate for different treatments and only three pregnancies were exposed to Anakinra [79]. Autoimmune diseases often have negative impact on fertility and pregnancy outcomes $[79,80]$. Two factors can be considered to affect the course of pregnancy, the disease or the treatment for this disease. In the exposed pregnancies included in this review, the indications for treatment were CAPS (34.1\%), Familial Mediterranean Fever (FMF-33\%), and AOSD/SJIA (20.4\%). There is only one report, to our knowledge, of CAPS during pregnancy treated with medication other than those targeting the IL-1 system [81]. This study reported a rate of miscarriage of $30 \%$ as compared to $10 \%$ for CAPS-patients treated with Anakinra [81]. Only two studies reported pregnancy with those pathologies all exposed to Anakinra or Canakinumab, therefore it is difficult to distinguish the treatment effect to that from the inflammatory pathology itself $[78,81]$. There is only one report of untreated pregnancies with different autoimmune disease that the one that are reported in this study making the evaluation of the pathologies themselves difficult. In this study, the authors compared pregnancy with or without all kind of DMARDs and healthy pregnancy. However, this study did not discriminate for different treatments and only three pregnancies were exposed to Anakinra [82].

For patients with FMF, increased rates of miscarriage, premature rupture of membranes and low birth weight were observed compared to pregnant women without the disease [80]. In this study, $80 \%$ of women received Colchicine and none received antiinflammatory treatment [80]. Furthermore, one retrospective study by Ben-Chetrit et al., reported an elevated rate of spontaneous abortion in untreated FMF as opposed to those treated with Colchicine [83]. Unfortunately, Colchicine resistance is often observed in FMF patients and Anakinra is increasingly used to prevent flare-ups of the disease [73]. As for AOSD, 5 case reports and 17 pregnancies were reviewed by [84]. In this cohort, most women were exposed to corticosteroid with several reported adverse outcomes such as spontaneous miscarriage observed (9.1\%), premature delivery $(18.2 \%)$ and FGR $(9.1 \%)$. In the current systematic review, $3 / 14$ pregnancies complicated with AOSD ended in premature delivery which is comparable to that reported by Mok et al. In a study by Garcia-Fernandez et al., on women with SJIA, 20\% had preterm delivery [85], which is in contrast to the current work in which we observed no preterm delivery in SJIA with anti-IL-1 treatment. This difference could be explained by the treatment since most women in this systematic review received Anakinra as opposed to corticosteroid or other DMARDs.

We reported three miscarriages out of 88 pregnancies. All these losses occurred during the first trimester and two were exposed to Anakinra whereas one exposed to Canakinumab. This is in accordance with the literature that most miscarriages will occur during the first trimester; however, it is very difficult to measure the rate of miscarriages in the general population. Furthermore, in this cohort, two out of the three miscarriage occurs in the same 
women who had Cogan syndrome, a rare and severe autoimmune disease. In the literature, only eight cases of successful pregnancy with this disease have been reported [86-91]. Thus, it cannot be concluded that the therapy caused pregnancy loss in these women.

In the current work, the rate of preterm birth was $17.6 \%$ (all conditions combined) as opposed to a baseline of $11.1 \%$ [92]. However, the reported rate of preterm birth in a population with inflammatory disease is known to be higher; namely 13.6\% in FMF [80], $18.1 \%$ in AOSD [84] and 20\% in SJIA [85]. Although the rate of preterm birth are similar overall, it is important to keep in mind that there is no report of untreated pregnancies with those inflammatory pathologies. Only one study reported a preterm birth rate of $9 \%$ in pregnancy with inflammatory pathologies without DMARDs treatment. The maternal condition in this study were Sjögren syndrome, undifferentiated connective tissue disease (UTCD), systemic lupus erythematosus (SLE), antiphospholipid syndrome (APS) and others [82].

Our review reports neonatal complications in $13.6 \%$ of pregnancies exposed to Anakinra and $10.0 \%$ for those exposed to Canakinumab, totaling $13.2 \%$ who had complications overall. Of the 10 babies who had complications, six were diagnosed with CAPS whereas three had minor developmental delays or other problems and one died at 37 weeks (stillbirth). One baby of a FMF mother was hypotrophic, had respiratory distress syndrome and hyperbillirubilemia at birth; however, this baby was delivered prematurely at $33+5$ weeks and was healthy at 12 months of age [93]. Baby born preterm has more neonatal complication then their counterpart born at 37 week and onwards [94,95]. Another baby born to a mother with SJIA had right hydrocele and heart murmur at birth but these complications could be due to maternal exacerbation of symptom such as oligohydramnios and hypertension. At the follow up, this baby had no major long-term complications nor malformations [96]. Finally, one baby born to a mother with active refractory AOSD had renal agenesis and ectopic neurohypophysis [97]. This is the second case of renal agenesis in Anakinra-exposed patient. The first case was in a mother diagnose with NOMID and it was a twin dichorionic-diamniotic pregnancy with fetal demise of one fetus with bilateral renal agenesis at 30 weeks. The surviving twin had no congenital abnormality and was born at 38.7 weeks. This case of congenital malformation could potentially be explained by the increased risk factor of renal tract abnormalities in twin birth as mentioned by the authors $[97,98]$. Furthermore, a study by Wiesel et al. reported that renal agenesis occurs in 58 of 709,030 live birth, significantly lower than 2 cases out of 88 pregnancies in this systematic review. One group has made a hypothesis that a link between uncontrolled maternal disease and renal abnormalities can occurs [96] but the potential link between renal malformation and IL-1 pathway should be the focus of future studies.

It is interesting to note that three studies, not included in the current systematic review, have evaluated 10 men who received $\mathrm{IL}-1$ blockade prior to conception, resulting in 13 pregnancies. In those studies, six men had CAPS, two AOSD, one SJIA and one FMF. Seven received Anakinra (100 mg/day) and three were treated with Canakinumab (150 mg/ 8 weeks) at the time of conception. No adverse effect on the child wellbeing were reported after paternal exposure to IL-1 blockage $[97,99,100]$.

This is the first systematic review to examine the effects of IL-1 blockade during pregnancy and we provide a summary of all pregnancies exposed to Anakinra or Canakinumab. Our study also highlighted the current lack of data and identified research gaps to be addressed, particularly the difference between the effects on pregnancy of the inflammatory pathology being treated as compared to the treatment itself. Our study was limited by the fact that abstracts from conferences were also included, in order to cover all exposed pregnancies, but some information were missing in relation to doses and outcomes in these abstracts. 


\section{Conclusions}

In conclusion, this review summarizes all the pregnancy exposed to Il-1 blockage and no major obstetrical and neonatal complication was reported. Il-1 blockage during pregnancy could be safe and beneficial in cases of pregnancy with inflammatory conditions.

Supplementary Materials: The following are available online at https:/ /www.mdpi.com/article/ 10.3390/jcm11010225/s1; Data S1: Search strategy used for this systematic review. Table S1: Search strategy used for this systematic review.

Author Contributions: Conceptualization and methodology, M.-E.B. and S.G.; data curation, M.E.B., V.G., K.H. and S.G.; writing original draft preparation, M.-E.B. and S.G., writing review and editing, M.-E.B., D.J.L.H., A.E.P.H. and S.G.; supervision, S.G. All authors have read and agreed to the published version of the manuscript.

Funding: This research received no external funding.

Institutional Review Board Statement: Not applicable.

Informed Consent Statement: Not applicable.

Conflicts of Interest: The authors declare no conflict of interest.

\section{References}

1. Aye, I.L.; Jansson, T.; Powell, T.L. Interleukin-1beta inhibits insulin signaling and prevents insulin-stimulated system A amino acid transport in primary human trophoblasts. Mol. Cell. Endocrinol. 2013, 381, 46-55. [CrossRef] [PubMed]

2. Bainbridge, S.A.; Roberts, J.M.; von Versen-Hoynck, F.; Koch, J.; Edmunds, L.; Hubel, C.A. Uric acid attenuates trophoblast invasion and integration into endothelial cell monolayers. Am. J. Physiol. Cell Physiol. 2009, 297, C440-C450. [CrossRef] [PubMed]

3. Bainbridge, S.A.; von Versen-Höynck, F.; Roberts, J.M. Uric acid inhibits placental system A amino acid uptake. Placenta 2009, 30 195-200. [CrossRef] [PubMed]

4. $\quad$ Lei, J.; Vermillion, M.S.; Jia, B.; Xie, H.; Xie, L.; McLane, M.W.; Sheffield, J.S.; Pekosz, A.; Brown, A.; Klein, S.L.; et al. IL-1 receptor antagonist therapy mitigates placental dysfunction and perinatal injury following Zika virus infection. JCI Insight 2019, 4, e122678. [CrossRef]

5. Mulla, M.J.; Myrtolli, K.; Potter, J.; Boeras, C.; Kavathas, P.B.; Sfakianaki, A.K.; Tadesse, S.; Norwitz, E.R.; Guller, S.; Abrahams, V.M. Uric acid induces trophoblast IL-1beta production via the inflammasome: Implications for the pathogenesis of preeclampsia. Am. J. Reprod. Immunol. 2011, 65, 542-548. [CrossRef]

6. Brien, M.E.; Duval, C.; Palacios, J.; Boufaied, I.; Hudon-Thibeault, A.A.; Nadeau-Vallee, M.; Vaillancourt, C.; Sibley, C.P.; Abrahams, V.M.; Jones, R.L.; et al. Uric Acid Crystals Induce Placental Inflammation and Alter Trophoblast Function via an IL-1-Dependent Pathway: Implications for Fetal Growth Restriction. J. Immunol. 2017, 198, 443-451. [CrossRef]

7. Depino, A.M. Perinatal inflammation and adult psychopathology: From preclinical models to humans. Semin. Cell Dev. Biol. 2018, 77, 104-114. [CrossRef]

8. Hagberg, H.; Gressens, P.; Mallard, C. Inflammation during fetal and neonatal life: Implications for neurologic and neuropsychiatric disease in children and adults. Ann. Neurol. 2012, 71, 444-457.

9. Van Vliet, E.O.; de Kieviet, J.F.; van der Voorn, J.P.; Been, J.V.; Oosterlaan, J.; van Elburg, R.M. Placental pathology and long-term neurodevelopment of very preterm infants. Am. J. Obstet. Gynecol. 2012, 206, e481-e487. [CrossRef]

10. Neiger, R. Long-Term Effects of Pregnancy Complications on Maternal Health: A Review. J. Clin. Med. 2017, 6, 76. [CrossRef]

11. Erlebacher, A. Immunology of the maternal-fetal interface. Annu. Rev. Immunol. 2013, 31, 387-411. [CrossRef]

12. Moffett, A.; Loke, C. Immunology of placentation in eutherian mammals. Nat. Rev. Immunol. 2006, 6, 584-594. [CrossRef]

13. Mor, G.; Cardenas, I.; Abrahams, V.; Guller, S. Inflammation and pregnancy: The role of the immune system at the implantation site. Ann. N. Y. Acad. Sci. 2011, 1221, 80-87. [CrossRef]

14. Menon, R.; Richardson, L.S.; Lappas, M. Fetal membrane architecture, aging and inflammation in pregnancy and parturition. Placenta 2019, 79, 40-45. [CrossRef]

15. Romero, R.; Espinoza, J.; Gonçalves, L.F.; Kusanovic, J.P.; Friel, L.A.; Nien, J.K. Inflammation in preterm and term labour and delivery. Semin. Fetal Neonatal Med. 2006, 11, 317-326. [CrossRef]

16. Brien, M.E.; Boufaied, I.; Bernard, N.; Forest, J.C.; Giguere, Y.; Girard, S. Specific inflammatory profile in each pregnancy complication: A comparative study. Am. J. Reprod. Immunol. 2020, 84, e13316. [CrossRef]

17. Salazar Garcia, M.D.; Mobley, Y.; Henson, J.; Davies, M.; Skariah, A.; Dambaeva, S.; Gilman-Sachs, A.; Beaman, K.; Lampley, C.; Kwak-Kim, J. Early pregnancy immune biomarkers in peripheral blood may predict preeclampsia. J. Reprod. Immunol. 2018, 125, 25-31. [CrossRef]

18. Freeman, D.J.; McManus, F.; Brown, E.A.; Cherry, L.; Norrie, J.; Ramsay, J.E.; Clark, P.; Walker, I.D.; Sattar, N.; Greer, I.A. Shortand long-term changes in plasma inflammatory markers associated with preeclampsia. Hypertension 2004, 44, 708-714. [CrossRef] 
19. Ferguson, K.K.; Meeker, J.D.; McElrath, T.F.; Mukherjee, B.; Cantonwine, D.E. Repeated measures of inflammation and oxidative stress biomarkers in preeclamptic and normotensive pregnancies. Am. J. Obstet. Gynecol. 2017, 216, e521-e527. [CrossRef]

20. Redman, C.W.; Staff, A.C. Preeclampsia, biomarkers, syncytiotrophoblast stress, and placental capacity. Am. J. Obstet. Gynecol. 2015, 213, S9.e1-S9.e4. [CrossRef]

21. Taylor, B.D.; Ness, R.B.; Klebanoff, M.A.; Zoh, R.; Bass, D.; Hougaard, D.M.; Skogstrand, K.; Haggerty, C.L. First and second trimester immune biomarkers in preeclamptic and normotensive women. Pregnancy Hypertens. 2016, 6, 388-393. [CrossRef]

22. Taylor, B.D.; Tang, G.; Ness, R.B.; Olsen, J.; Hougaard, D.M.; Skogstrand, K.; Roberts, J.M.; Haggerty, C.L. Mid-pregnancy circulating immune biomarkers in women with preeclampsia and normotensive controls. Pregnancy Hypertens. 2016, 6, 72-78. [CrossRef]

23. Ronzoni, S.; Steckle, V.; D'Souza, R.; Murphy, K.E.; Lye, S.; Shynlova, O. Cytokine Changes in Maternal Peripheral Blood Correlate With Time-to-Delivery in Pregnancies Complicated by Premature Prelabor Rupture of the Membranes. Reprod. Sci. 2018, 26, 1266-1276. [CrossRef]

24. Giguere, Y.; Masse, J.; Theriault, S.; Bujold, E.; Lafond, J.; Rousseau, F.; Forest, J.C. Screening for pre-eclampsia early in pregnancy: Performance of a multivariable model combining clinical characteristics and biochemical markers. BJOG 2015, 122, $402-410$. [CrossRef]

25. Kuc, S.; Wortelboer, E.J.; van Rijn, B.B.; Franx, A.; Visser, G.H.; Schielen, P.C. Evaluation of 7 serum biomarkers and uterine artery Doppler ultrasound for first-trimester prediction of preeclampsia: A systematic review. Obstet. Gynecol. Surv. 2011, 66, 225-239. [CrossRef]

26. Yu, N.; Cui, H.; Chen, X.; Chang, Y. First trimester maternal serum analytes and second trimester uterine artery Doppler in the prediction of preeclampsia and fetal growth restriction. Taiwan J. Obstet. Gynecol. 2017, 56, 358-361. [CrossRef]

27. Bianchi, M.E. DAMPs, PAMPs and alarmins: All we need to know about danger. J. Leukoc. Biol. 2007, 81, 1-5. [CrossRef]

28. Matzinger, P. The danger model: A renewed sense of self. Science 2002, 296, 301-305. [CrossRef]

29. Brien, M.E.; Baker, B.; Duval, C.; Gaudreault, V.; Jones, R.L.; Girard, S. Alarmins at the maternal-fetal interface: Involvement of inflammation in placental dysfunction and pregnancy complications (1). Can. J. Physiol. Pharmacol. 2019, 97, 206-212. [CrossRef]

30. Nadeau-Vallee, M.; Obari, D.; Palacios, J.; Brien, M.E.; Duval, C.; Chemtob, S.; Girard, S. Sterile inflammation and pregnancy complications: A review. Reproduction 2016, 152, R277-R292. [CrossRef]

31. Sharps, M.C.; Baker, B.C.; Guevara, T.; Bischof, H.; Jones, R.L.; Greenwood, S.L.; Heazell, A.E.P. Increased placental macrophages and a pro-inflammatory profile in placentas and maternal serum in infants with a decreased growth rate in the third trimester of pregnancy. Am. J. Reprod. Immunol. 2020, 84, e13267. [CrossRef] [PubMed]

32. Saji, F.; Samejima, Y.; Kamiura, S.; Sawai, K.; Shimoya, K.; Kimura, T. Cytokine production in chorioamnionitis. J. Reprod. Immunol. 2000, 47, 185-196. [CrossRef]

33. Russo, R.C.; Garcia, C.C.; Teixeira, M.M. Anti-inflammatory drug development: Broad or specific chemokine receptor antagonists? Curr. Opin. Drug Discov. Dev. 2010, 13, 414-427.

34. Grainger, D.J.; Reckless, J. Broad-spectrum chemokine inhibitors (BSCIs) and their anti-inflammatory effects in vivo. Biochem. Pharmacol. 2003, 65, 1027-1034. [CrossRef]

35. Bandoli, G.; Palmsten, K.; Forbess Smith, C.J.; Chambers, C.D. A Review of Systemic Corticosteroid Use in Pregnancy and the Risk of Select Pregnancy and Birth Outcomes. Rheum. Dis. Clin. N. Am. 2017, 43, 489-502. [CrossRef]

36. Shanks, A.L.; Grasch, J.L.; Quinney, S.K.; Haas, D.M. Controversies in antenatal corticosteroids. Semin. Fetal Neonatal Med. 2019, 24, 182-188. [CrossRef]

37. Wapner, R.J.; Sorokin, Y.; Mele, L.; Johnson, F.; Dudley, D.J.; Spong, C.Y.; Peaceman, A.M.; Leveno, K.J.; Malone, F.; Caritis, S.N.; et al. Long-term outcomes after repeat doses of antenatal corticosteroids. N. Engl. J. Med. 2007, 357, 1190-1198. [CrossRef]

38. Bandyopadhyay, A.; Slaven, J.E.; Evrard, C.; Tiller, C.; Haas, D.M.; Tepper, R.S. Antenatal corticosteriods decrease forced vital capacity in infants born fullterm. Pediatr. Pulmonol. 2020, 55, 2630-2634. [CrossRef]

39. Bérard, A.; Sheehy, O.; Girard, S.; Zhao, J.P.; Bernatsky, S. Risk of preterm birth following late pregnancy exposure to NSAIDs or COX-2 inhibitors. Pain 2018, 159, 948-955. [CrossRef]

40. Daniel, S.; Matok, I.; Gorodischer, R.; Koren, G.; Uziel, E.; Wiznitzer, A.; Levy, A. Major malformations following exposure to nonsteroidal antiinflammatory drugs during the first trimester of pregnancy. J. Rheumatol. 2012, 39, 2163-2169. [CrossRef]

41. Kenyon, S.; Pike, K.; Jones, D.R.; Brocklehurst, P.; Marlow, N.; Salt, A.; Taylor, D.J. Childhood outcomes after prescription of antibiotics to pregnant women with spontaneous preterm labour: 7-year follow-up of the ORACLE II trial. Lancet 2008, 372, 1319-1327. [CrossRef]

42. Kenyon, S.; Pike, K.; Jones, D.R.; Brocklehurst, P.; Marlow, N.; Salt, A.; Taylor, D.J. Childhood outcomes after prescription of antibiotics to pregnant women with preterm rupture of the membranes: 7-year follow-up of the ORACLE I trial. Lancet 2008, 372, 1310-1318. [CrossRef]

43. Muanda, F.T.; Sheehy, O.; Bérard, A. Use of antibiotics during pregnancy and risk of spontaneous abortion. CMAJ 2017, 189, E625-E633. [CrossRef]

44. Muanda, F.T.; Sheehy, O.; Bérard, A. Use of antibiotics during pregnancy and the risk of major congenital malformations: A population based cohort study. Br. J. Clin. Pharmacol. 2017, 83, 2557-2571. [CrossRef] 
45. Reis, A.S.; Barboza, R.; Murillo, O.; Barateiro, A.; Peixoto, E.P.M.; Lima, F.A.; Gomes, V.M.; Dombrowski, J.G.; Leal, V.N.C.; Araujo, F.; et al. Inflammasome activation and IL-1 signaling during placental malaria induce poor pregnancy outcomes. Sci. Adv. 2020, 6, eaax6346. [CrossRef]

46. Equils, O.; Kellogg, C.; McGregor, J.; Gravett, M.; Neal-Perry, G.; Gabay, C. The role of the IL-1 system in pregnancy and the use of IL-1 system markers to identify women at risk for pregnancy complications. Biol. Reprod. 2020, 103, 684-694. [CrossRef]

47. Southcombe, J.H.; Redman, C.W.; Sargent, I.L.; Granne, I. Interleukin-1 family cytokines and their regulatory proteins in normal pregnancy and pre-eclampsia. Clin. Exp. Immunol. 2015, 181, 480-490. [CrossRef]

48. Licini, C.; Tossetta, G.; Avellini, C.; Ciarmela, P.; Lorenzi, T.; Toti, P.; Gesuita, R.; Voltolini, C.; Petraglia, F.; Castellucci, M.; et al. Analysis of cell-cell junctions in human amnion and chorionic plate affected by chorioamnionitis. Histol. Histopathol. 2016, 31, 759-767.

49. Tossetta, G.; Paolinelli, F.; Avellini, C.; Salvolini, E.; Ciarmela, P.; Lorenzi, T.; Emanuelli, M.; Toti, P.; Giuliante, R.; Gesuita, R.; et al. IL-1 $\beta$ and TGF- $\beta$ weaken the placental barrier through destruction of tight junctions: An in vivo and in vitro study. Placenta 2014, 35, 509-516. [CrossRef]

50. Girard, S.; Heazell, A.E.; Derricott, H.; Allan, S.M.; Sibley, C.P.; Abrahams, V.M.; Jones, R.L. Circulating cytokines and alarmins associated with placental inflammation in high-risk pregnancies. Am. J. Reprod. Immunol. 2014, 72, 422-434. [CrossRef]

51. Girard, S.; Sébire, H.; Brochu, M.E.; Briota, S.; Sarret, P.; Sébire, G. Postnatal administration of IL-1Ra exerts neuroprotective effects following perinatal inflammation and/or hypoxic-ischemic injuries. Brain Behav. Immun. 2012, 26, 1331-1339. [CrossRef]

52. Girard, S.; Tremblay, L.; Lepage, M.; Sebire, G. IL-1 receptor antagonist protects against placental and neurodevelopmental defects induced by maternal inflammation. J. Immunol. 2010, 184, 3997-4005. [CrossRef]

53. Leitner, K.; Al Shammary, M.; McLane, M.; Johnston, M.V.; Elovitz, M.A.; Burd, I. IL-1 receptor blockade prevents fetal cortical brain injury but not preterm birth in a mouse model of inflammation-induced preterm birth and perinatal brain injury. Am. $J$. Reprod. Immunol. 2014, 71, 418-426. [CrossRef]

54. Nadeau-Vallee, M.; Chin, P.Y.; Belarbi, L.; Brien, M.E.; Pundir, S.; Berryer, M.H.; Beaudry-Richard, A.; Madaan, A.; Sharkey, D.J.; Lupien-Meilleur, A.; et al. Antenatal Suppression of IL-1 Protects against Inflammation-Induced Fetal Injury and Improves Neonatal and Developmental Outcomes in Mice. J. Immunol. 2017, 198, 2047-2062. [CrossRef]

55. Nadeau-Vallee, M.; Quiniou, C.; Palacios, J.; Hou, X.; Erfani, A.; Madaan, A.; Sanchez, M.; Leimert, K.; Boudreault, A.; Duhamel, F; et al. Novel Noncompetitive IL-1 Receptor-Biased Ligand Prevents Infection- and Inflammation-Induced Preterm Birth. J. Immunol. 2015, 195, 3402-3415. [CrossRef]

56. McDuffie, R.S., Jr.; Davies, J.K.; Leslie, K.K.; Lee, S.; Sherman, M.P.; Gibbs, R.S. A randomized controlled trial of interleukin-1 receptor antagonist in a rabbit model of ascending infection in pregnancy. Infect. Dis. Obstet. Gynecol. 2001, 9, 233-237. [CrossRef]

57. Presicce, P.; Park, C.W.; Senthamaraikannan, P.; Bhattacharyya, S.; Jackson, C.; Kong, F.; Rueda, C.M.; DeFranco, E.; Miller, L.A.; Hildeman, D.A.; et al. IL-1 signaling mediates intrauterine inflammation and chorio-decidua neutrophil recruitment and activation. JCI Insight 2018, 3, e98306. [CrossRef]

58. Karisnan, K.; Bakker, A.J.; Song, Y.; Noble, P.B.; Pillow, J.J.; Pinniger, G.J. Interleukin-1 receptor antagonist protects against lipopolysaccharide induced diaphragm weakness in preterm lambs. PLoS ONE 2015, 10, e0124390.

59. Nadeau-Vallee, M.; Obari, D.; Quiniou, C.; Lubell, W.D.; Olson, D.M.; Girard, S.; Chemtob, S. A critical role of interleukin-1 in preterm labor. Cytokine Growth Factor Rev. 2016, 28, 37-51. [CrossRef]

60. Prieto-Peña, D.; Dasgupta, B. Biologic agents and small-molecule inhibitors in systemic autoimmune conditions: An update. Pol. Arch. Intern. Med. 2020, 131, 171-181. [CrossRef]

61. Soh, M.C.; Moretto, M. The use of biologics for autoimmune rheumatic diseases in fertility and pregnancy. Obstet. Med. 2020, 13, 5-13. [CrossRef] [PubMed]

62. Götestam Skorpen, C.; Hoeltzenbein, M.; Tincani, A.; Fischer-Betz, R.; Elefant, E.; Chambers, C.; da Silva, J.; Nelson-Piercy, C.; Cetin, I.; Costedoat-Chalumeau, N.; et al. The EULAR points to consider for use of antirheumatic drugs before pregnancy, and during pregnancy and lactation. Ann. Rheum. Dis. 2016, 75, 795-810. [CrossRef] [PubMed]

63. Nuki, G.; Bresnihan, B.; Bear, M.B.; McCabe, D. Long-term safety and maintenance of clinical improvement following treatment with anakinra (recombinant human interleukin-1 receptor antagonist) in patients with rheumatoid arthritis: Extension phase of a randomized, double-blind, placebo-controlled trial. Arthritis Rheum. 2002, 46, 2838-2846. [CrossRef] [PubMed]

64. Buckley, L.F.; Viscusi, M.M.; Van Tassell, B.W.; Abbate, A. Interleukin-1 blockade for the treatment of pericarditis. Eur. Heart J. Cardiovasc. Pharmacother. 2018, 4, 46-53. [CrossRef]

65. Kary, S.; Burmester, G.R. Anakinra: The first interleukin-1 inhibitor in the treatment of rheumatoid arthritis. Int. J. Clin. Pract. 2003, 57, 231-234.

66. Ramírez, J.; Cañete, J.D. Anakinra for the treatment of rheumatoid arthritis: A safety evaluation. Expert Opin. Drug Saf. 2018, 17, 727-732. [CrossRef]

67. Dinarello, C.A.; van der Meer, J.W. Treating inflammation by blocking interleukin-1 in humans. Semin. Immunol. 2013, 25, 469-484. [CrossRef]

68. Vastert, S.J.; Jamilloux, Y.; Quartier, P.; Ohlman, S.; Osterling Koskinen, L.; Kullenberg, T.; Franck-Larsson, K.; Fautrel, B.; de Benedetti, F. Anakinra in children and adults with Still's disease. Rheumatology 2019, 58, vi9-vi22. [CrossRef]

69. Church, L.D.; McDermott, M.F. Canakinumab, a fully-human mAb against IL-1beta for the potential treatment of inflammatory disorders. Curr. Opin. Mol. Ther. 2009, 11, 81-89. 
70. Savic, S.; McDermott, M.F. Inflammation: Canakinumab for the cryopyrin-associated periodic syndromes. Nat. Rev. Rheumatol. 2009, 5, 529-530. [CrossRef]

71. Ortona, E.; Pierdominici, M.; Maselli, A.; Veroni, C.; Aloisi, F.; Shoenfeld, Y. Sex-based differences in autoimmune diseases. Ann. Ist. Super. Sanita 2016, 52, 205-212.

72. Fischer-Betz, R.; Specker, C. Pregnancy in systemic lupus erythematosus and antiphospholipid syndrome. Best Pract. Res. Clin. Rheumatol. 2017, 31, 397-414. [CrossRef]

73. Ugurlu, S.; Ergezen, B.; Egeli, B.H.; Selvi, O.; Ozdogan, H. Anakinra treatment in patients with familial Mediterranean fever: A single-centre experience. Rheumatology 2021, 60, 2327-2332. [CrossRef]

74. Moher, D.; Liberati, A.; Tetzlaff, J.; Altman, D.G. Preferred reporting items for systematic reviews and meta-analyses: The PRISMA statement. PLoS Med. 2009, 6, e1000097. [CrossRef]

75. Sterne, J.A.; Hernán, M.A.; Reeves, B.C.; Savović, J.; Berkman, N.D.; Viswanathan, M.; Henry, D.; Altman, D.G.; Ansari, M.T.; Boutron, I.; et al. ROBINS-I: A tool for assessing risk of bias in non-randomised studies of interventions. BMJ 2016, 355 , i4919. [CrossRef]

76. Murad, M.H.; Sultan, S.; Haffar, S.; Bazerbachi, F. Methodological quality and synthesis of case series and case reports. BMJ Evid.-Based Med. 2018, 23, 60-63. [CrossRef]

77. Sfriso, P.; Bindoli, S.; Doria, A.; Feist, E.; Galozzi, P. Canakinumab for the treatment of adult-onset Still's disease. Expert Rev. Clin. Immunol. 2020, 16, 129-138. [CrossRef]

78. Egawa, M.; Imai, K.; Mori, M.; Miyasaka, N.; Kubota, T. Placental Transfer of Canakinumab in a Patient with Muckle-Wells Syndrome. J. Clin. Immunol. 2017, 37, 339-341. [CrossRef]

79. Mijatovic, V.; Hompes, P.G.; Wouters, M.G. Familial Mediterranean fever and its implications for fertility and pregnancy. Eur. J. Obstet. Gynecol. Reprod. Biol. 2003, 108, 171-176. [CrossRef]

80. Yasar, O.; Iskender, C.; Kaymak, O.; Taflan Yaman, S.; Uygur, D.; Danisman, N. Retrospective evaluation of pregnancy outcomes in women with familial Mediterranean fever. J. Matern. Fetal Neonatal Med. 2014, 27, 733-736. [CrossRef]

81. Chang, Z.; Spong, C.Y.; Jesus, A.A.; Davis, M.A.; Plass, N.; Stone, D.L.; Chapelle, D.; Hoffmann, P.; Kastner, D.L.; Barron, K.; et al. Anakinra use during pregnancy in patients with cryopyrin-associated periodic syndromes (CAPS). Arthritis Rheum. 2014, 66, 3227-3232. [CrossRef]

82. De Lorenzo, R.; Ramirez, G.A.; Punzo, D.; Lorioli, L.; Rovelli, R.; Canti, V.; Barera, G.; Rovere-Querini, P. Neonatal outcomes of children born to mothers on biological agents during pregnancy: State of the art and perspectives. Pharmacol. Res. 2020, 152, 104583. [CrossRef]

83. Ben-Chetrit, E.; Ben-Chetrit, A.; Berkun, Y.; Ben-Chetrit, E. Pregnancy outcomes in women with familial Mediterranean fever receiving colchicine: Is amniocentesis justified? Arthritis Care Res. 2010, 62, 143-148. [CrossRef]

84. Mok, M.Y.; Lo, Y.; Leung, P.Y.; Lau, C.S. Pregnancy outcome in patients with adult onset Still's disease. J. Rheumatol. 2004, 31, 2307-2309.

85. García-Fernández, A.; Gerardi, M.C.; Crisafulli, F.; Filippini, M.; Fredi, M.; Gorla, R.; Lazzaroni, M.G.; Lojacono, A.; Nalli, C.; Ramazzotto, F.; et al. Disease course and obstetric outcomes of pregnancies in juvenile idiopathic arthritis: Are there any differences among disease subtypes? A single-centre retrospective study of prospectively followed pregnancies in a dedicated pregnancy clinic. Clin. Rheum. 2021, 40, 239-244. [CrossRef]

86. Bakalianou, K.; Salakos, N.; Iavazzo, C.; Danilidou, K.; Papadias, K.; Kondi-Pafiti, A. A rare case of uneventful pregnancy in a woman with Cogan's syndrome. Clin. Exp. Obstet. Gynecol. 2008, 35, 301-302.

87. Currie, C.; Wax, J.R.; Pinette, M.G.; Blackstone, J.; Cartin, A. Cogan's syndrome complicating pregnancy. J. Matern. Fetal Neonatal Med. 2009, 22, 928-930. [CrossRef]

88. Deliveliotou, A.; Moustakarias, T.; Argeitis, J.; Vaggos, G.; Vitoratos, N.; Hassiakos, D. Successful full-term pregnancy in a woman with Cogan's syndrome: A case report. Clin. Rheum. 2007, 26, 2181-2183. [CrossRef]

89. Riboni, F.; Cosma, S.; Perini, P.G.; Benedetto, C. Successful Pregnancy in a Patient with Atypical Cogan's Syndrome. Isr. Med. Assoc. J. 2016, 18, 495-496.

90. Tarney, C.M.; Wilson, K.; Sewell, M.F. Cogan syndrome in pregnancy. Obstet. Gynecol. 2014, 124, 428-431. [CrossRef]

91. Venhoff, N.; Thiel, J.; Schramm, M.A.; Jandova, I.; Voll, R.E.; Glaser, C. Case Report: Effective and Safe Treatment with Certolizumab Pegol in Pregnant Patients With Cogan's Syndrome: A Report of Three Pregnancies in Two Patients. Front. Immunol. 2020, 11, 616992. [CrossRef] [PubMed]

92. Blencowe, H.; Cousens, S.; Chou, D.; Oestergaard, M.; Say, L.; Moller, A.B.; Kinney, M.; Lawn, J. Born too soon: The global epidemiology of 15 million preterm births. Reprod. Health 2013, 10 (Suppl. 1), S2. [CrossRef] [PubMed]

93. Venhoff, N.; Voll, R.E.; Glaser, C.; Thiel, J. IL-1-blockade with Anakinra during pregnancy: Retrospective analysis of efficacy and safety in female patients with familial Mediterranean fever. Z. Rheum. 2018, 77, 127-134. [CrossRef] [PubMed]

94. Romero, R.; Dey, S.K.; Fisher, S.J. Preterm labor: One syndrome, many causes. Science 2014, 345, 760-765. [CrossRef]

95. Saigal, S.; Doyle, L.W. An overview of mortality and sequelae of preterm birth from infancy to adulthood. Lancet 2008, 371, 261-269. [CrossRef]

96. Smith, C.J.F.; Chambers, C.D. Five successful pregnancies with antenatal anakinra exposure. Rheumatology 2018, 57, 1271-1275. [CrossRef] 
97. Youngstein, T.; Hoffmann, P.; Gül, A.; Lane, T.; Williams, R.; Rowczenio, D.M.; Ozdogan, H.; Ugurlu, S.; Ryan, J.; Harty, L.; et al. International multi-centre study of pregnancy outcomes with interleukin-1 inhibitors. Rheumatology 2017, 56, 2102-2108. [CrossRef]

98. Rider, R.A.; Stevenson, D.A.; Rinsky, J.E.; Feldkamp, M.L. Association of twinning and maternal age with major structural birth defects in Utah, 1999 to 2008. Birth Defects Res. A Clin. Mol. Teratol. 2013, 97, 554-563. [CrossRef]

99. Viktil, K.K.; Engeland, A.; Furu, K. Use of antirheumatic drugs in mothers and fathers before and during pregnancy-a populationbased cohort study. Pharmacoepidemiol. Drug Saf. 2009, 18, 737-742. [CrossRef]

100. Drechsel, P.; Stüdemann, K.; Niewerth, M.; Horneff, G.; Fischer-Betz, R.; Seipelt, E.; Spähtling-Mestekemper, S.; Aries, P.; Zink, A.; Klotsche, J.; et al. Pregnancy outcomes in DMARD-exposed patients with juvenile idiopathic arthritis-results from a JIA biologic registry. Rheumatology 2020, 59, 603-612. [CrossRef] 